\title{
Estimation of edge electron temperature profiles via forward modelling of the electron cyclotron radiation transport at ASDEX Upgrade
}

\author{
S K Rathgeber ${ }^{1}$, L Barrera ${ }^{1}$, T Eich ${ }^{1}, \mathbf{R}$ Fischer $^{1}$, B Nold ${ }^{2}$, \\ W Suttrop ${ }^{1}, M$ Willensdorfer ${ }^{3}, \mathrm{E} \mathrm{Wolfrum}^{1}$ and the ASDEX \\ Upgrade Team \\ 1 Max-Planck-Institut für Plasmaphysik, EURATOM Association, \\ Boltzmannstraße 2, 85748 Garching, Germany \\ 2 Institut für Plasmaforschung, Universität Stuttgart, \\ 70569 Stuttgart, Germany \\ ${ }^{3}$ Institut für angewandte Physik, Technische Universität Wien, Association \\ EURATOM-ÖAW, A-1040 Vienna, Austria \\ E-mail: sylvia.rathgeber@ipp.mpg.de
}

\begin{abstract}
.
We present a method to obtain reliable edge profiles of the electron temperature by forward modelling of the electron cyclotron radiation transport. While for the core of ASDEX Upgrade plasmas, straightforward analysis of electron cyclotron intensity measurements based on the optically thick plasma approximation is usually justified, reasonable analysis of the steep and optically thin plasma edge needs to consider broadened emission and absorption profiles and radiation transport processes. This is done in the framework of integrated data analysis which applies Bayesian probability theory for joint analysis of the electron density and temperature with data of different interdependent and complementary diagnostics.

By this means, electron cyclotron radiation intensity delivers highly spatially resolved electron temperature data for the plasma edge. In H-mode, the edge gradient of the electron temperature can be several times higher than the one of the radiation temperature. Furthermore, we are able to reproduce the 'shine-through' peak - the observation of increased radiation temperatures at frequencies resonant in the optically thin scrape-off layer. This phenomenon is caused by strongly down-shifted radiation of Maxwellian tail electrons located in the $\mathrm{H}$-mode edge region and, therefore, contains valuable information about the electron temperature edge gradient.
\end{abstract}

\section{Introduction}

For the realization of a fusion reactor, one of the most crucial tasks is the understanding and control of the physical processes occurring at the plasma edge. The latter determines quality and stability of the plasma confinement [1]. Within this very thin layer some plasma parameters vary by several orders of magnitude. Therefore, detailed knowledge with high temporal and spatial resolution of the plasma edge characteristics, e.g. 
the electron density and temperature for ELM studies and investigations on the L$\mathrm{H}$ transition, is essential for the development of future fusion plants. In this work, we present a method to obtain reliable edge profiles of the electron temperature by forward modelling of the electron cyclotron radiation transport.

Radiometry of the electron cyclotron intensity spectrum is an established technique to diagnose the electron temperature profile [2]. Nevertheless, its commonly employed analysis is not universally applicable. It is based on the identification of the radiation temperature measured at a certain frequency with the local electron temperature at the corresponding cold resonance position [3]. The underlying assumptions of discretely localized emission and of radiation intensity at black-body level are reasonable in the core of typical fusion plasmas. However, they are no longer valid for frequencies that are resonant in optically thin plasma regions or close to steep temperature gradients, e.g. at the edge of H-mode plasmas.

A direct evidence for the misinterpretation of identifying the radiation temperature with the electron temperature is given by the so-called 'shine-through' peak of enhanced radiation at frequencies resonant in the Scrape-Off Layer (SOL) which can reach several hundred $\mathrm{eV}$ and has been observed at different machines $[4,5,6]$. Such high electron temperatures outside the separatrix would cause an immediate plasma extinction due to the strong parallel heat transport $\left(K_{\|} \propto T^{5 / 2}\right)[7]$.

This implies, that establishing reliable electron temperature profiles at the plasma edge needs handling of the radiation transport processes through the entire plasma and consideration of the broadened electron cyclotron emission and absorption profiles which depend on the electron temperature and density [8]. This is done by forward modelling in the framework of Integrated Data Analysis (IDA) which combines the data of LIthium Beam emission spectroscopy (LIB), Deuterium CyaNide interferometry (DCN) and Electron Cyclotron Emission (ECE) diagnostic for joint analysis of the electron density and temperature, applying Bayesian probability theory [9].

For H-mode plasmas, the presented method reveals steeper edge electron temperature gradients than previous analysis. This finding changes the basis of plasma edge studies, since the pressure gradient — a key player for pedestal physics — is strongly dependent on the temperature gradient.

Furthermore, our analysis is able to quantitatively reproduce the measured electron cyclotron radiation intensities for all frequencies resonant in the main and near-SOL plasma. This also includes the 'shine-through' peak which can be modelled — in contradiction to previous thoughts $[4,5]$ — without the inclusion of any non-Maxwellian contributions to the electron distribution function.

The article consists of two methods and four results sections followed by a discussion. The methods part starts with a short overview on the diagnostic setup and hardware installed at ASDEX Upgrade for electron cyclotron intensity measurements. Section 3 is dedicated to the physical background of ECE data and its analysis. First, some basics about electron cyclotron radiation and its transport together with the required mathematics are introduced and a new formula providing an analytical 
solution for the broadened emission profile of second harmonic eXtraordinary mode $\left(2^{\text {nd }}\right.$ harmonic X-mode) waves caused by Maxwell-distributed electrons is presented. Second, the principles of the optimization process within the preexisting IDA environment for electron density and temperature estimation at ASDEX Upgrade are pointed out. Finally, section 3.3 describes in detail how the modelling of the electron cyclotron radiation is realized within IDA.

After presenting typical results for the H-mode edge electron temperature obtained with our new method in section 4, we explain the relation between the electron temperature and the radiation temperature subject to different plasma characteristics in section 5. The sensitivity of our results on program settings and input parameters is studied in section 6 and the method is validated in section 7 .

\section{ECE measurement at ASDEX Upgrade}

At ASDEX Upgrade, the electron cyclotron intensity spectrum in $2^{\text {nd }}$ harmonic $\mathrm{X}$ mode is measured with a 60-channel heterodyne radiometer receiver [2]. It is equipped with five mixers at local oscillator frequencies of $\nu_{\mathrm{LO}}=95,101,128,133$ and $167 \mathrm{GHz}$. Together with 60 filters at fixed intermediate frequencies in the range of $\nu_{\mathrm{IF}}=2-18 \mathrm{GHz}$ with bandwidths of $300 \mathrm{MHz}$ for 36 channels and $600 \mathrm{MHz}$ for 24 channels this defines the sensitivity range for the measured radio frequencies $\left(\nu_{\mathrm{RF}}=85-185 \mathrm{GHz}\right)$ and the instrumental radial resolution of down to $\sim 5 \mathrm{~mm}$. The data acquisition system exhibits a temporal resolution of $32 \mu \mathrm{s}$. The system is absolutely calibrated by measurements of black-body radiation emitted by laboratory hot $(T=773 \mathrm{~K})$ and cold sources $(T=77 \mathrm{~K})$ [2].

The ECE antennae are located slightly above the midplane of the ASDEX Upgrade vessel, viewing the plasma from the low-field side. The lines of sight (LOS) are formed by a system of three lenses that cause Gaussian beams focussed on the plasma edge. They are oriented towards the toroidal magnetic field with an angle $\theta+\theta_{r}$. The angle between the magnetic field lines and the central rays ranges between $\theta \approx 96^{\circ}-100^{\circ}$ for one and $\theta \approx 80^{\circ}-85^{\circ}$ for the other antennae, while $\theta_{r}$ which accounts for the widened viewing area due to the finite antenna pattern lies within $\pm 5^{\circ}$.

\section{Electron cyclotron radiation forward modelling}

\subsection{Electron cyclotron radiation}

Details about the origin of electron cyclotron radiation, its intensity and transport via (re-)emission and re-absorption processes can be found i.a. in [8]. However, we introduce the formulae needed for the analysis and sketch the derivation of a new analytical formula for the broadened emission profile. Furthermore, the assumptions and insufficiency of previous ECE analysis are discussed. 
Gyration frequency Due to their gyration around the magnetic field lines the electrons in a magnetically confined fusion plasma emit electromagnetic radiation at the fundamental electron cyclotron frequency and its harmonics $m$. The cold resonance angular frequencies

$$
\omega_{m}=m \frac{e B}{m_{\mathrm{e}, 0}}
$$

are determined by the magnetic field strength $B$ and the electron rest mass $m_{\mathrm{e}, 0}$ and charge $e$. For every single electron, the actual emission frequencies are broadened around the discrete cold resonance frequencies as a result of its individual relativistic mass increase and the Doppler effect [8].

Emission profile An expression for this broadened emission profile considering the freespace approximation for sufficiently tenuous plasma and single uncorrelated radiating electrons is derived e.g. in [8]. A weakly relativistic plasma $\left(k_{\mathrm{B}} T_{\mathrm{e}} \ll m_{\mathrm{e}, 0} c^{2}\right)$ in local thermal equilibrium (Maxwell-distributed electrons) yields for the $2^{\text {nd }}$ harmonic X-mode $\left(\omega_{m}=\omega_{2 \mathrm{X}}=2 e B / m_{\mathrm{e}, 0}\right)$ a spectral emissivity of:

$$
j_{2 \mathrm{X}}(\omega)=\eta_{2 \mathrm{X}} j \Phi(\omega)
$$

with total emissivity

$$
j=\left(\frac{e \omega_{2 \mathrm{X}}}{4 \pi \zeta}\right)^{2} \frac{n_{\mathrm{e}}}{\epsilon_{0} c} \sin ^{2} \theta\left(\cos ^{2} \theta+1\right),
$$

normalized $\left(\int \Phi(\omega) d \omega=1\right)$ shape function

$\Phi(\omega)=\frac{\zeta^{7 / 2}}{\sqrt{\pi}} \int \delta\left(\left[1-\beta_{\|} \cos \theta\right] \omega-\sqrt{1-\beta^{2}} \omega_{2 \mathrm{x}}\right) \exp \left(-\zeta\left[\beta_{\perp}^{2}+\beta_{\|}^{2}\right]\right) \beta_{\perp}^{5} \mathrm{~d} \beta_{\perp} \mathrm{d} \beta_{\|}$

and the fraction of power that is emitted in X-mode $\eta_{2 \mathrm{X}}\left(=1-\eta_{2 \mathrm{O}}\right) . \quad \beta_{\|}=v_{\|} / c$ and $\beta_{\perp}=v_{\perp} / c$ denote the parallel and perpendicular velocity contributions as a fraction of the speed of light $c, \theta$ the viewing angle relative to the magnetic field lines and $\zeta=\left(m_{\mathrm{e}, 0} c^{2}\right) /\left(2 k_{\mathrm{B}} T_{\mathrm{e}}\right)$ with Boltzmann constant $k_{\mathrm{B}}$ and electron temperature $T_{\mathrm{e}}$. From the delta function, giving only a contribution to the emissivity if the resonance condition

$$
\omega=\frac{\sqrt{1-\beta_{\|}^{2}-\beta_{\perp}^{2}}}{1-\beta_{\|} \cos \theta} \omega_{2 \mathrm{X}}
$$

is fulfilled, one can derive the broadening of the emission region depending on electron velocity and viewing angle. Doppler broadening is dominant if $\beta_{\|} \cos \theta>\beta^{2}$. Elsewhere the frequency is mainly down-shifted relative to the cold resonance frequency due to relativistic mass increase. [8]

The integration of (3.4) is carried out in (A.1) and reveals an analytic result for the shape function:

$$
\begin{aligned}
& \Phi(\omega)=\frac{\zeta^{3 / 2}}{\sqrt{\pi} \omega \mu \cos ^{5} \theta} \times \\
& \times\left[\exp (-\zeta\{1-\mu \alpha\})\left(-\frac{\sin ^{2} \theta}{\sqrt{\alpha}}+\frac{\eta^{2}}{\zeta \mu}-\frac{1}{\sqrt{\zeta \mu}}\left\{3 \eta-2 \zeta \mu \sin ^{2} \theta\right\} F(\sqrt{\zeta \mu \alpha})\right)\right]_{\alpha_{2}}^{\alpha_{1}}
\end{aligned}
$$


with substitutions $\mu=\omega^{2} / \omega_{2 \mathrm{X}}^{2}, \alpha=\left(1-\beta_{\|} \cos \theta\right)^{2}$ and $\eta=1+\mu \cos ^{2} \theta$ and Dawson integral $F(x)=\exp \left(-x^{2}\right) \int_{0}^{x} \exp \left(y^{2}\right) \mathrm{d} y$. The bounds of integration are given by $\alpha_{1,2}=\left(1 \pm \sqrt{1-\mu \sin ^{2} \theta} \cos \theta\right)^{2} / \eta^{2}$.

Radiation transport In a fusion plasma the magnetic field strength varies with the location and, therefore, radiation at a certain frequency is emitted from a finite resonance region according to the broadening effects. Hence, to obtain the radiation intensity $I_{2 \mathrm{X}}$ emerging the plasma, emission and re-absorption processes - corrected by the refractive index $N$ - have to be considered within the whole broadened resonance region via the radiation transport equation [3]:

$\frac{\mathrm{d}}{\mathrm{d} s} \frac{I_{2 \mathrm{X}}(s)}{N^{2}(s)}=\frac{1}{N^{2}(s)}\left(j_{2 \mathrm{X}}(s)-\alpha_{2 \mathrm{X}}(s) I_{2 \mathrm{X}}(s)\right)=\frac{j_{2 \mathrm{X}}(s)}{N^{2}(s)}\left(1-\frac{I_{2 \mathrm{X}}(s)}{I_{\mathrm{BB}, 2 \mathrm{X}}(s)}\right)$

along the ray path $s$. In local thermal equilibrium, the absorption $\alpha_{2 \mathrm{X}}$ is thereby given by Kirchhoff's law. $I_{\mathrm{BB}}$ denotes the black-body intensity which for hot plasmas and electron cyclotron radiation in the microwave range $\left(\hbar \omega<m e V \ll k_{\mathrm{B}} T_{\mathrm{e}}\right)$ is given by the Rayleigh-Jeans approximation

$$
I_{\mathrm{BB}}(\omega)=\frac{\omega^{2}}{8 \pi^{3} c^{2}} k_{\mathrm{B}} T_{\mathrm{e}}
$$

of Planck's radiation formula [8].

'Classical' ECE analysis Commonly applied ECE analysis (hereafter: 'classical' ECE analysis) simplifies the radiation transport by assuming an optically thick plasma with optical depth $\tau=\int \alpha(s) \mathrm{d} s \gg 1$. In this case, the incident radiation is fully reabsorbed after sufficient path length and - according to (3.7) and under stationary plasma conditions - the intensity $I_{2 \mathrm{X}}(s)$ is constant along the path and equal to blackbody intensity [3]. As a second effect of the high re-absorption, the emission region of the radiation actually emerging the plasma is narrowed and the origin of the main intensity contribution is localized closer to the position of cold resonance. 'Classical' ECE analysis consequently applies the approximation of a plasma emitting electron cyclotron radiation at black-body level from the cold resonance position $R_{\text {res }}$ of the measured frequency $\omega$. In this case the analysis is straight-forward since the electron temperature - equally to the radiation temperature $T_{\text {rad }}$ - is directly proportional to the measured intensity:

$$
T_{\mathrm{e}}\left(R_{\mathrm{res}}(\omega)\right)=\frac{8 \pi^{3} c^{2}}{k_{\mathrm{B}} \omega^{2}} I=T_{\text {rad }}(\omega) .
$$

For mostly observed first harmonic Ordinary mode $\left(1^{\text {st }}\right.$ harmonic O-mode $)$ and $2^{\text {nd }}$ harmonic X-mode, the assumption of an optically thick plasma is usually valid in the bulk of a fusion plasma [8,3]. Anyway, in regions of steep temperature gradients and optically thin plasma - typical edge conditions for H-mode plasmas - the 'classical' ECE analysis fails and the broadened emission and absorption profiles have to be considered. 


\subsection{Integrated data analysis}

For the estimation of the electron temperature from measured electron cyclotron radiation intensities, we applied the concept of IDA in the framework of Bayesian probability theory. Bayes' theorem

$$
p(x, y \mid d)=\frac{p(d \mid x, y) p(x, y)}{p(d)}
$$

enables to link the likelihood Probability Density Functions (PDFs) $p(d \mid x, y)$, denoting the conditional probability for the measured data set $d$ with given parameter values $x, y$ for the quantities of interest, of different diagnostics together with the prior PDFs $p(x, y)$, containing a priori information about the physical quantities. The product of all likelihood and prior PDFs — both usually initialized as Gaussian or Cauchy distributions - is proportional to the posterior $\operatorname{PDF} p(x, y \mid d)$ which provides the joint probability distribution for the quantities of interest considering all the underlying measured data from various diagnostics simultaneously. Maximization of the posterior PDF delivers the parameter values that are most likely to produce the measured data set. $[10,11]$

At ASDEX Upgrade, IDA is routinely used for the estimation of electron density and temperature profiles including LIB and DCN data for the density and ECE data for the temperature evaluation as presented in [9].

The error analysis applies the $\chi^{2}$-binning method [12] where single or groups of neighboured density/temperature values are increased and decreased as long as the modelled data $D_{i}$ of channel $i$ deteriorates from the measured data $d_{i, j}$ at time $j$ such that $\Delta \chi^{2}=\Delta \sum_{i, j}\left(\left[d_{i, j}-D_{i}\right] / \Delta d_{i, j}\right)^{2}>1$. These values form the upper and lower bounds of the original value. This rather simple method with independent error bars that do not include any prior information provides an intuitive assessment of the informational content of the measurement.

\subsection{Code implementation}

Our newly developed Electron Cyclotron radiation Forward Modelling (ECFM) code replaces the assumption of the 'classical' ECE analysis which has previously been implemented in the IDA program. It generates the radiation temperature for given electron density and temperature profiles by modelling the electron cyclotron radiation transport. The surrounding IDA process (cf. left-hand side of figure 1), of forming the likelihood PDFs for every diagnostic, combining them via Bayes' theorem with the prior PDFs, maximizing the posterior PDF via gradient-based optimization processes and assessing the uncertainties via $\chi^{2}$-binning method, is - apart from the inclusion of some additional prior information — applied as described in [9].

Input data The scheme of the ECFM code is sketched on the right-hand side of figure 1. It needs complete profiles of the electron density and temperature as input. These are provided as exponentials of cubic B-splines over several parameter values at different positions between $\rho=0$ and 1.2. The normalised poloidal radius 


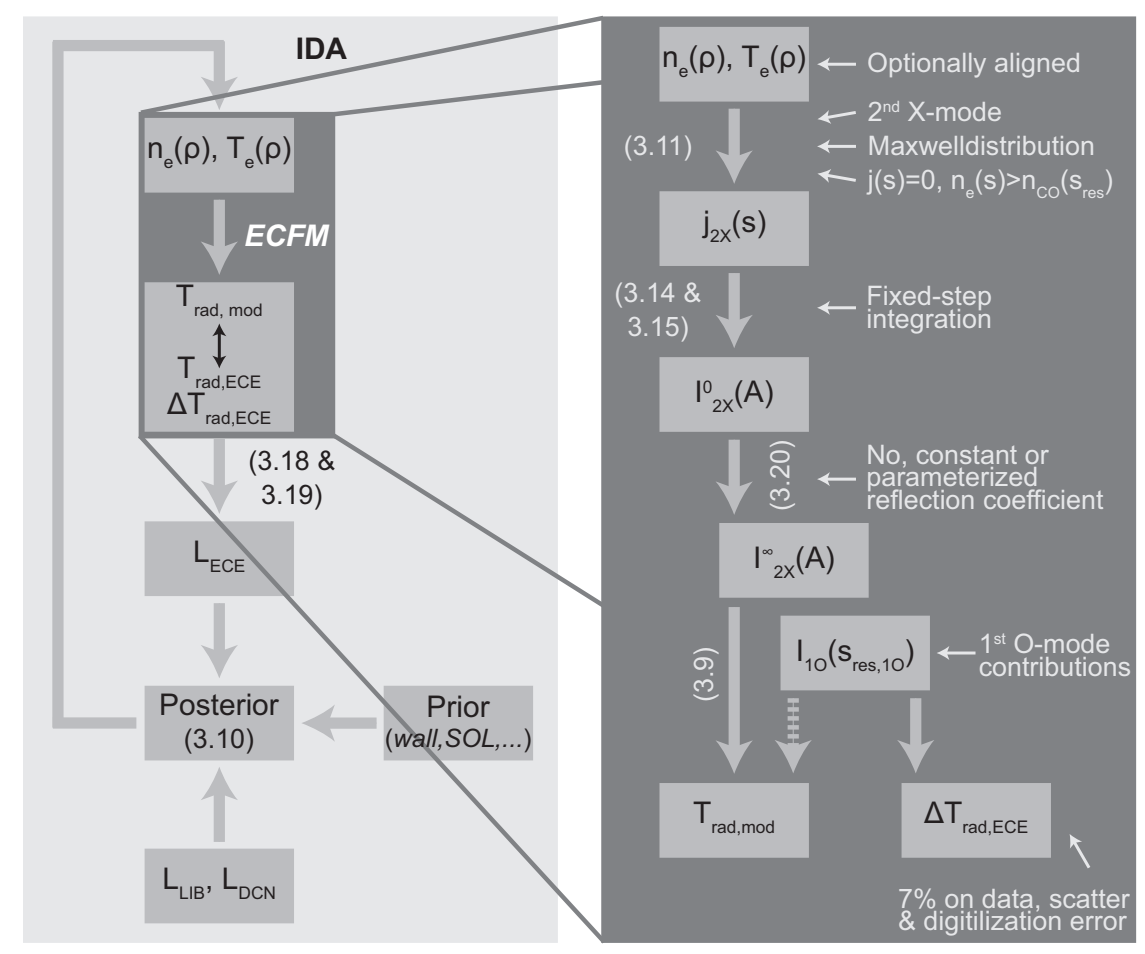

Figure 1. Left: Scheme of the IDA process; the new features (ECFM code and additional prior information) are indicated by italics, right: Scheme of the ECFM code; the solid arrows mark the default process, the numbers denote the equation used for the corresponding step in the program

$\rho=\sqrt{\left(\Psi-\Psi_{\text {axis }}\right) /\left(\Psi_{\text {separatrix }}-\Psi_{\text {axis }}\right)}$ with poloidal flux $\Psi$ offers a common coordinate system for all the diagnostics. The density profile can optionally be aligned to the temperature profile. This alignment can be regulated manually by a fixed radial scale of the density profile or automatically by adapting the position of the density value with maximum curvature - which is assumed to occur at the separatrix [13] - either to $\rho=1.0$ or to the position of $T_{\mathrm{e}}=100 \mathrm{eV}$ (default setting) - a reasonable separatrix condition for the electron temperature [14]. Apart from the alignment, the density profile is mainly determined by the LIB and DCN data and is only slightly adapted to the ECE data.

Calculation of the radiation intensity Given $n_{\mathrm{e}}(\rho), T_{\mathrm{e}}(\rho)$ and their mapping to the ECE viewing lines, the emissivity

$$
j_{\nu, r}(s)= \begin{cases}0 & \text { for } n_{\mathrm{e}}(s)>n_{\mathrm{co}, 2 \mathrm{X}}(s) \\ \eta_{2 \mathrm{X}}(s) j(s) \Phi_{\nu, r}(s) & \text { else }\end{cases}
$$

is calculated at every position $s$ along the LOS where the density is lower than the cut-off density $n_{\mathrm{co}, 2 \mathrm{X}}=2 \epsilon_{0} m_{\mathrm{e}, 0}\left(\pi \nu_{2 \mathrm{X}} / e\right)^{2}$ of the $2^{\text {nd }}$ harmonic X-mode [15] and set to 0 elsewhere, because no radiation from inside the cut-off layer can contribute. Possible reflections are considered separately as described later. ECE channels that are in cut off 
at the position of their cold resonance are discarded completely. The X-mode fraction $\eta_{2 \mathrm{X}}(s)$ of $(3.11)$ is determined by

$$
\eta_{2 \mathrm{x}}=\frac{1}{2}+\frac{\frac{1}{8} \sin ^{4} \theta+\cos ^{2} \theta}{\left(\cos ^{2} \theta+1\right) \sqrt{\cos ^{2} \theta+\frac{1}{16} \sin ^{4} \theta}}
$$

applying the tenuous plasma approach $\left(\nu \gg \nu_{\mathrm{p}}\right)$ which is usually fulfilled at the plasma edge [8] and, therefore, appropriate for our purpose. The total emissivity $j(s)$ and the shape function $\Phi_{\nu, r}(s)$ are given by (3.3) and (3.6). Here, it should be noted that we switched from angular frequency $\omega$ to the measured frequency $\nu=\omega /(2 \pi)$. To increase the radial resolution not only the emission profile of the central frequency $\nu_{\mathrm{ECE}}$ of each channel is calculated, but its measured bandwidth $\Delta \nu_{\mathrm{ECE}}$ is discretized in $\mathrm{N}_{\nu}$ (Default: $\mathrm{N}_{\nu}=3$ ) equally distributed frequencies with bandwidths $\Delta \nu=\Delta \nu_{\mathrm{ECE}} / \mathrm{N}_{\nu}$. At the same time, the LOS can be split up in $\mathrm{N}_{r}$ toroidally distributed rays $r$, accounting for the finite beam width. The electron density and temperature values along each ray are thereby taken over from the central ray of the corresponding LOS. This can be motivated by the rays each transversing plasma regions with the same characteristics, given the validity of toroidal symmetry. Therefore, to consider the antenna pattern only the angle to the magnetic field has to be adapted. Due to the negligible curvature of the magnetic field lines within the small viewed area this is done by simply adding/subtracting the angle between the examined and the central ray $\theta_{r}$ to/from the angle between the central ray and the magnetic field lines $\theta: \angle(\vec{B}$, ray $)=\theta \pm \theta_{r}$.

Knowing the emissivity and via Kirchhoff's law also the absorption, the intensity for each frequency and each ray is obtained by solving the radiation transport equation with the assumption of a refractive index of $N=1$ in the tenuous plasma approach:

$$
\frac{\mathrm{d} I_{\nu}(s)}{\mathrm{d} s}=j_{\nu}(s)-\alpha_{\nu}(s) I_{\nu}(s)
$$

This is done by simple integration with $N_{\mathrm{s}}=1000$ fixed steps $\Delta s(\approx 0.1 \mathrm{~mm}$ close to the cold resonance position and $\approx 2 \mathrm{~mm}$ elsewhere) from the inner wall to the antenna:

$$
I_{\nu, r}(\mathrm{~A})=\sum_{\mathrm{W}}^{\mathrm{A}}\left(j_{\nu, r}(s)-\alpha_{\nu, r}(s) I_{\nu, r}(s)\right) \Delta s .
$$

From the computational point of view it would be preferable to use an ODE solver with adaptable step size to solve the radiation transport equation. Unfortunately, the emissivity calculation is not absolutely numerically stable because the terms in (3.6) can possess very similar values which leads to large round-off errors. This can cause discontinuities in the intensity calculation which are way too small to have an influence on the result, but are not tolerable for the gradient-based optimization process.

To get the total modelled single-pass radiation intensity $I_{\mathrm{A}}^{0}\left(\nu_{\mathrm{ECE}}\right)$ for one ECE channel, one has to sum up all the discretized frequencies and rays

$$
I_{\nu_{\mathrm{ECE}}}^{0}(\mathrm{~A})=\sum_{\nu=1}^{\mathrm{N}_{\nu}} \sum_{r=1}^{\mathrm{N}_{r}} I_{\nu, r}(\mathrm{~A}) w\left(\theta_{r}\right) \Psi(\nu) \Delta \nu
$$


with the Gauss-shaped weighting function with full width of half maximum $\Delta \theta_{r}$ and the discretized antenna pattern $\mathrm{d} \theta_{r}$

$$
w\left(\theta_{r}\right)=\sqrt{\frac{\ln 2}{\pi}} \frac{2}{\Delta \theta_{\mathrm{r}}} \exp \left(-\frac{4 \ln 2}{\left(\Delta \theta_{\mathrm{r}}\right)^{2}} \theta_{r}^{2}\right) \mathrm{d} \theta_{r}
$$

for the different rays and with the filter function

$$
\Psi(\nu)= \begin{cases}\frac{1}{\Delta \nu_{\mathrm{ECE}}} & \text { for } \nu_{\mathrm{ECE}}-\frac{\Delta \nu_{\mathrm{ECE}}}{2} \leq \nu \leq \nu_{\mathrm{ECE}}+\frac{\Delta \nu_{\mathrm{ECE}}}{2} \\ 0 & \text { else }\end{cases}
$$

of the band pass filter.

Finally, the radiation intensity is translated into the radiation temperature $T_{\text {rad,mod }}\left(\nu_{\mathrm{ECE}}\right)$ via $(3.9)$ which is then compared to the measured data within the likelihood PDF

$$
\begin{aligned}
& \mathrm{L}_{\mathrm{ECE}}=\frac{1}{\prod_{i, j} \sqrt{2 \pi\left(\Delta T_{\mathrm{rad}, \mathrm{ECE}}(i, j)\right)^{2}}} \exp \left(-\frac{1}{2} \chi^{2}\right) \\
& \chi^{2}=\sum_{i, j}\left(\frac{T_{\mathrm{rad}, \mathrm{ECE}}(i, j)-T_{\mathrm{rad}, \mathrm{mod}}(i)}{\Delta T_{\mathrm{rad}, \mathrm{ECE}}(i, j)}\right)^{2}
\end{aligned}
$$

for all ECE channels $i$ and measured time points $j$ within the time frame selected for the analysis where the profiles are assumed to be constant.

Wall reflections Optionally, contributions from wall reflections and the $1^{\text {st }}$ harmonic Omode can be added to the modelled radiation intensity. The former might cause multiple ray passes through the plasma and - especially in the fully tungsten-coated ASDEX Upgrade machine - a significantly enhanced intensity $I_{\mathrm{A}}$ at the antenna compared to the single-pass intensity $I_{\mathrm{A}}^{0}$. This is incorporated within ECFM via the infinite-reflection model

$$
I_{\mathrm{A}}=\frac{I_{\mathrm{A}}^{0}}{1-\mathrm{Re}^{-\tau \mathrm{WA}}}
$$

considering infinite reflections back and forth between parallel walls with reflection coefficient $R$ for both walls [3]. In an optically thick plasma with optical depth between inner wall and antenna $\tau_{\text {WA }} \gg 1$ this does not cause a contribution to the antenna intensity, because the reflected intensity is re-absorbed completely, but it has to be taken into account in optically thin plasmas. The reflection coefficient is either given by a fixed value (default: $R=0.99$ ) or as a model parameter.

$1^{\text {st }}$ harmonic O-mode contributions Due to harmonic overlap and mode conversion by polarization scrambling at the walls, radiation emitted by electrons at the high-field side in $1^{\text {st }}$ harmonic O-mode might influence the measurement. These contributions are usually negligible compared to the $2^{\text {nd }}$ harmonic X-mode radiation emitted in the main plasma since they are mostly re-absorbed on their path through the optically thick 
plasma. Furthermore, densities in typical ASDEX Upgrade discharges are generally much higher than the cut-off density of the $1^{\text {st }}$ harmonic O-mode. Anyway, it is conceivable that O-mode radiation emitted outside the cut-off layer on the high-field side travels poloidally around the plasma by reflections back and forth between the inner vessel wall and the cut-off layer and reaches the antenna at the low-field side - partly converted to X-mode. This can hardly be modelled due to the complex reflection structures and unknown depolarization fraction. However, $1^{\text {st }}$ harmonic Omode contributions can be roughly approximated by the application of the optically thick plasma approach and the assumption that they are fully converted to X-mode and completely reach the antenna: $T_{\text {rad }}^{\mathrm{tot}}\left(\nu_{\mathrm{ECE}}\right)=T_{\mathrm{rad}}^{2 \mathrm{X}}\left(\nu_{\mathrm{ECE}}\right)+T_{\mathrm{rad}}^{1 \mathrm{O}}\left(\nu_{\mathrm{ECE}}\right)=T_{\mathrm{rad}}^{2 \mathrm{X}}\left(\nu_{\mathrm{ECE}}\right)+$ $T_{\mathrm{e}}\left(\rho_{\mathrm{res}, 1 \mathrm{O}}\right)$. This approach has been used to estimate the sensitivity of the model to the $1^{\text {st }}$ harmonic O-mode contributions. Anyway, since it is generally not valid, possible O-mode contributions are usually not considered in the calculation of the absolute radiation temperature value, but only taken into account as an additional source of uncertainty in the measurement. Outside a certain value (default: $\rho=1.05$ ), where Omode contributions are assumed to dominate over radiation emitted in the $2^{\text {nd }}$ harmonic $\mathrm{X}$-mode, the ECE data are given very large error bars and, therefore, are neglected in the model.

Error assessment Additionally to these contributions, the uncertainty in the ECE measurement is composed of an approximated error of $7 \%$ on the data including systematic uncertainties from the calibration process and the temperature dependence of the gains and the mixers, plus the $1 \sigma$-standard deviation of the statistical scatter within the analyzed time frame, plus a digitization error of $15 \mathrm{eV}\left(\Delta T_{\text {rad,dat }}=7 \% T_{\text {rad,dat }}+\sigma+\right.$ $\left.15 \mathrm{eV}\left(+100 \% \mathrm{~T}_{\text {rad,mod }}^{1 \mathrm{O}}\right)\right)$. The latter was introduced to account for uncertainties that might arise in the low intensity edge channels since the current data acquisition system does not allow optimized gain settings, but uses a fixed gain for every channel.

A priori information Additionally to the prior information of former standard IDA application, we included two prior PDFs that penalize high electron temperature values in the SOL (default: $\left.T_{\mathrm{e}}(\rho>1.02) \ngtr 50 \mathrm{eV}\right)$ and outside the first vessel wall (default: $\left.T_{\mathrm{e}}\left(\rho>\rho_{\mathrm{W}}\right) \ngtr 2 \mathrm{eV}\right)$. The latter acts like an outer boundary for the extrapolation in the region with omitted or without ECE data. The SOL prior is sometimes necessary to avoid bifurcation in the modelling of the 'shine-through' peak. If it is not too pronounced and the SOL density sufficiently high, the 'shine-through' peak can not only be modelled by radiation shining through from the hotter plasma edge region, but also by very hot electrons located in the SOL. The suppression of the latter solution is justified assuming a typical ASDEX Upgrade separatrix temperature of $T_{\mathrm{e}, \mathrm{sep}}=100 \mathrm{eV}$ and power decay length of $\lambda_{q}=3 \mathrm{~mm}$ [18] which results in an electron temperature decay length of $\lambda_{T_{\mathrm{e}}} \approx 10 \mathrm{~mm}$ according to Spitzer-Härm conductivity and even less in the flux-limited model for parallel heat conduction [19] and, therefore, in an electron temperature $T_{\mathrm{e}}(\rho>1.02)<37 \mathrm{eV}$. 
Computational time Regarding its computational time, our ECFM code is not yet optimized for routine analysis. Each calculation of the complete radiation temperature profile which means more or less solving (3.15) for all $\mathrm{N}_{\mathrm{ch}}=60$ ECE channels with default values of $\mathrm{N}_{\nu}=3$ and $\mathrm{N}_{r}=1$ takes approximately $0.13 \mathrm{~s}$. This value is increased by several orders of magnitude within the optimization process and error analysis of IDA such that the time consumption of a complete IDA run for one time point is in the order of $10 \mathrm{~min}$. The most time consuming part of the forward model is given by the calculation of the emission shape function (3.6) which is done $\mathrm{N}_{\text {ch }} \times \mathrm{N}_{\nu} \times \mathrm{N}_{\mathrm{r}} \times \mathrm{N}_{\mathrm{s}}=1.8 \times 10^{5}$ times. Due to the linear dependence of the computational time on $\mathrm{N}_{\nu}$ and $\mathrm{N}_{r}$ and their weak influence on the results (cf. section 6) we only use a small number of frequency and ray discretizations for standard analysis. This finding demonstrates the importance of our new analytical formula for the emission profile which fastens its calculation by orders of magnitude.

\section{Edge electron temperature profiles in $\mathrm{H}$-mode discharges}

Since the H-mode serves as baseline scenario for fusion power plants and at the same time exhibits — with its steep edge gradients - the strong need for a sophisticated ECE analysis like ECFM to obtain reliable edge electron temperatures, we exemplify the results of an arbitrary ASDEX Upgrade standard H-mode discharge \#25804 and an H-mode discharge with strongly pronounced 'shine-through' peak \#23417. Both discharges were performed with plasma current of $I_{\mathrm{P}}=1.0 \mathrm{MA}$ and toroidal magnetic field $B_{\mathrm{t}}=-2.5 \mathrm{~T}$. Discharge $\# 25804$ was heated with $P=9.1 \mathrm{MW}$ and exhibited a central electron density $n_{\mathrm{e}, 0}=8.4 \times 10^{19} \mathrm{~m}^{-3}$ at the analyzed time point $t=2.06 \mathrm{~s}$. The total heating power and central density of discharge $\# 23417$ at $t=3.47 \mathrm{~s}$ were lower with $P=6.6 \mathrm{MW}$ and $n_{\mathrm{e}, 0}=8.0 \times 10^{19} \mathrm{~m}^{-3}$.

Figure 2(a) and (e) show the edge electron temperature profiles (red lines) obtained via ECFM analysis. Displayed by red pluses are the corresponding modelled radiation temperature values at the positions of their cold resonances. For both discharges they agreed very well with the ECE measurements (black crosses) up to the near SOL region — including the 'shine-through' peak.

The consistency between modelled and measured data was also reflected in the residuals $\operatorname{res}_{\mathrm{i}, \mathrm{j}}=\left(T_{\mathrm{rad}, \mathrm{ECE}}(i, j)-T_{\mathrm{rad}, \bmod }(i)\right) / \Delta T_{\mathrm{rad}, \mathrm{ECE}}(i, j)$ - stating the deviance between the modelled and measured values weighted by the measurement uncertainties. All residual values in figure $2(\mathrm{~b})$ and (f) lie between \pm 1 which means that the modelled values matched the measurement within its uncertainty. Outside of $\rho=1.02$ for $\# 25804$ and $\rho=1.05$ for $\# 23417$ the ECE data was assumed to contain a significant amount of $1^{\text {st }}$ harmonic O-mode contributions and, therefore, was omitted in the analysis and its uncertainty set to a very large value. This caused residual values close to zero. The large error bars at $\rho \approx 0.83$ and $\rho \approx 0.93$ in figure $2(\mathrm{a})$ and at $\rho<0.87$ in (e) arose from the large gap between the measured data while for $\rho>1$ in (e) they demonstrate that the ECE measurement did not contain sufficient information for a precise estimation of 

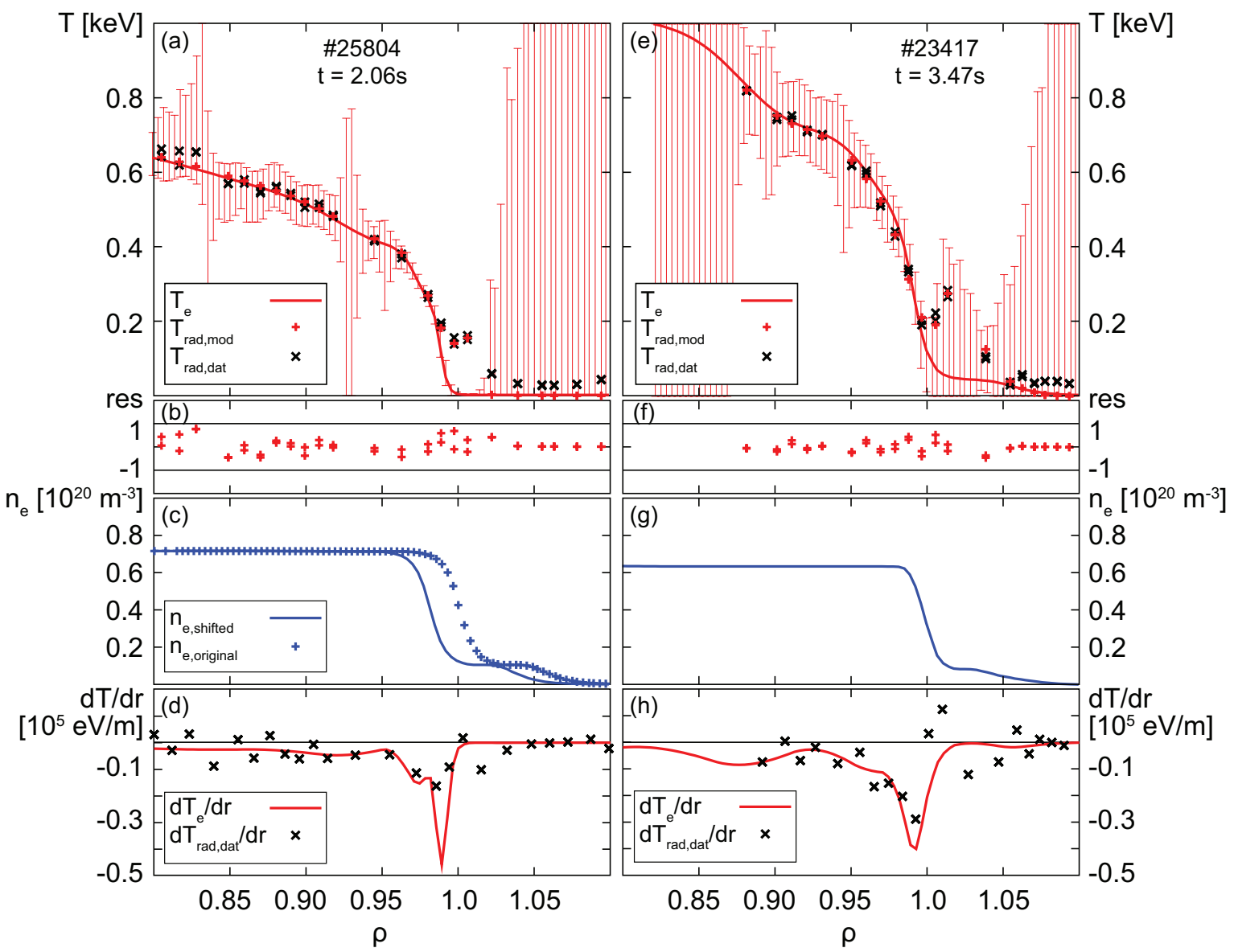

Figure 2. (a,e) Edge $T_{\mathrm{e}}$ profiles (red lines) together with the modelled (red pluses) and measured (black crosses) $T_{\text {rad }}$ values at the positions of their cold resonance, (b,f) their residuals, (c,g) the applied (line) and original (pluses) $n_{\mathrm{e}}$ profile and (d,h) the gradients of $T_{\mathrm{e}}$ (red lines) and $T_{\text {rad }}$ (black crosses) of discharge $\# 25804$ at $t=2.06 \mathrm{~s}$ (left) and \#23417 at $t=3.47 \mathrm{~s}$ (right)

the electron temperature in the SOL. The explanation for this will be given in the next section.

Panels (c) and (g) additionally depict the electron density used for the forward modelling (solid lines). For ECFM analysis of discharge \#25804 the electron density profile had been shifted according to the separatrix condition mentioned in section 3.3.

The most important observation for the electron temperature results was their increased steepness compared to the radiation temperature and, therefore, also compared to the electron temperature results from 'classical' analysis. For discharge \#25804 the maximal electron temperature gradient was more then two times higher than the radiation temperature gradient (cf. figure 2(d)), for discharge \#23417 the maximum of the electron temperature gradient exceeded the maximum of the radiation temperature gradient by around $40 \%$ (cf. figure $2(\mathrm{~h})$ ). 


\section{Radiation temperature versus electron temperature in H-mode discharges}

The scope of this section is to explain the large deviations between the electron and radiation temperature and their gradients at the edge and in the SOL. In the following we show qualitatively for different regions with characteristic plasma conditions regarding optical depth and gradient strength how the radiation temperature behaves compared to the electron temperature at its cold resonance position. Therefore, the emission and absorption profiles and the radiation transport of three representative ECE channels with cold resonances marked by vertical black lines in figure 3 are described in detail.

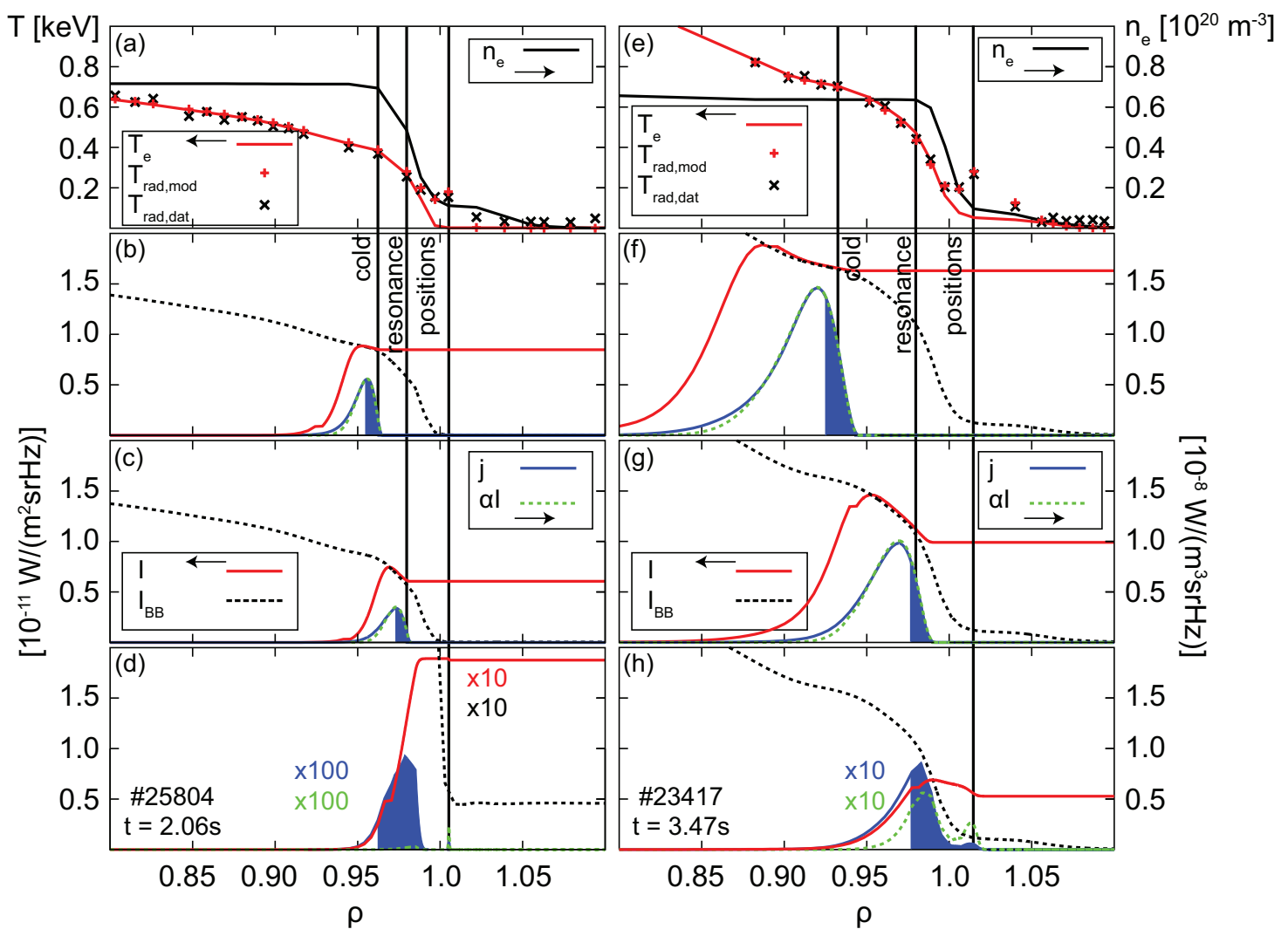

Figure 3. (a,e) Edge $T_{\mathrm{e}}$ profiles (red lines) together with the modelled (red pluses) and measured (black crosses) $T_{\text {rad }}$ values at the positions of their cold resonance and the applied $n_{\mathrm{e}}$ profiles (black lines). Line integrated radiation (red, solid) and black-body intensity (black, dashed) together with the corresponding emission (blue, solid) and absorption (green, dashed) profiles of ECE channel with mean frequency (b) $\nu_{E C E}=110.4 \mathrm{GHz}$, (c) $\nu_{E C E}=109.8 \mathrm{GHz}$, (d) $\nu_{E C E}=108.9 \mathrm{GHz}$, (f) $\nu_{E C E}=114.4 \mathrm{GHz},(\mathrm{g}) \nu_{E C E}=112.9 \mathrm{GHz}$ and (h) $\nu_{E C E}=111.8 \mathrm{GHz}$, with their marked resonance positions (vertical black lines) and actual emission origins (filled blue range) for discharge $\# 25804$ at $t=2.06 \mathrm{~s}$ (left) and $\# 23417$ at $t=3.47 \mathrm{~s}$ (right)

Optically thick and low gradient plasma region For discharge \#25804 the ECE channel with central frequency of $\nu_{\mathrm{ECE}}=110.4 \mathrm{GHz}$ had its cold resonance at $\rho=0.96$. In 
this region of high electron density and temperature, already after a short ray path, the whole emitted radiation (blue, solid in figure $3(\mathrm{~b})$ ) was fully re-absorbed (green, dashed) and the electron cyclotron intensity (red, solid) reached black-body level (black, dashed). At the same time, the high re-absorption narrowed the region in which the actual measured radiation has been emitted (filled range). The determination of the actual emission region was done by back-integration of the radiation transport from the antenna towards the plasma center until the modelled radiation intensity was reached. In this case of high optical depth and low gradients the actually viewed region was located around the position of the cold resonance and exhibited only small changes in electron density and temperature. Under these conditions, the modelled radiation temperature (red plus in figure 3(a)) matched the electron temperature (red line) at the cold resonance position of the measured frequency at $\rho=0.96$ as predicted by the 'classical' ECE analysis described in section 3.1. The same behaviour was observed for ECE channel with central frequency of $\nu_{\mathrm{ECE}}=114.4 \mathrm{GHz}$ of discharge $\# 23417$ (cf. figure $3(\mathrm{f}))$.

Optically thick and high gradient plasma region The cold resonance positions of frequencies $\nu_{\mathrm{ECE}}=109.8 \mathrm{GHz}(\# 25804)$ and $\nu_{\mathrm{ECE}}=112.9 \mathrm{GHz}(\# 23417)$ illustrated in figure $3(\mathrm{c})$ and (g) were located near the pedestal top where the plasma was still optically thick but close to the steep edge. Again, the high absorption assured radiation at blackbody level and a narrow emission region around the cold resonance position. However, the emission region reached the steep gradient area with large variances in the radiation level. In case of discharge \#25804 the main contribution to the antenna intensity came from slightly inside the cold resonance position which resulted in a radiation temperature higher than the electron temperature at the cold resonance position. In case of discharge \#23417 the main emission origin was shifted slightly outwards into regions with rapidly falling electron density and temperature which resulted in a radiation temperature smaller than the electron temperature at the cold resonance position.

Optically thin and high gradient plasma region The resonance regions of the ECE channels with central frequencies $\nu_{\mathrm{ECE}}=108.9 \mathrm{GHz}(\# 25804)$ and $\nu_{\mathrm{ECE}}=111.8 \mathrm{GHz}$ (\#23417) were optically thin (incomplete re-absorption) and the observation areas remained rather broad (figure $3(\mathrm{~d})$ and $(\mathrm{h})$ ). In fact, the main contribution of the measured radiation was emitted far inside the cold resonance position in the much hotter edge gradient region which caused a radiation temperature which greatly exceeded the actual electron temperature at its cold resonance position - the 'shine-through' peak.

This also explains the large uncertainty of the SOL electron temperature in figure 2(e). The radiation contribution from the SOL was too low as to provide significant information on the local electron temperature. However, since the 'shinethrough' peak contained information about the edge gradient region it allowed to reduce the uncertainty of the electron temperature in this very important region. The low error bars around the separatrix in figure 2 (a) do not indicate that the informational content 
from this area was larger than for discharge \#23417 because its emission contribution was also negligible. However, larger SOL electron temperatures would have increased the absorption such that the 'shine-through' peak could not have been modelled anymore. This might be an indication for an overestimated electron density.

\section{Sensitivity of the electron temperature result on the model inputs}

Due to non-linear physics (e.g. electron temperature and density dependence of the emission profile) and non-quantifiable sources of systematic uncertainties (e.g. wall reflections) of the different diagnostics, IDA incorporates high complexity and a large amount of degrees of freedom. This might cause non-transparent responses of the model to its inputs. This section is, therefore, dedicated to a detailed sensitivity study that we performed to demonstrate the influence of some model inputs on the ECFM output which on the one hand improved the understanding of the interaction between the model and its inputs and on the other hand allowed to narrow the area of validity of uncertain input parameters.

We identified the numerical or physical origin of the respective behaviour and were able to find objective criteria for proper selection of program settings. As a quantitative measure for the quality of the modelling, we considered $\chi^{2}$ given by $(3.19)$ or $\chi_{\mathrm{n}}^{2}$ normalized on the number of ECE channels and the number of measurement points per analyzed time point. It should be noted here, that - in contrast to considering statistically distributed uncertainties only - in our case, $\chi_{\mathrm{n}}^{2}$ laid below 1 since systematic uncertainties were also taken into account. The dominant contribution to the uncertainty of the radiation temperature was made of $7 \%$ on the data (cf. section 3.3) which was correlated for all channels. This strongly reduced $\chi_{\mathrm{n}}^{2}$. In addition, the inclusion of data points that were neglected in the analysis by a very large uncertainty caused a negligible contribution to $\chi^{2}$ while they were still weighted and, therefore, reduced the normalized $\chi^{2}$ further.

\subsection{Sensitivity on the number of spline knots}

The first sensitivity study accounted for the number and position of spline knots that were used to parameterize the electron temperature profile. In our investigation the nodes between $\rho=0-0.8$ were kept fixed, while the number of nodes at the plasma edge was increased with either equidistant distribution (crosses in figure 4 left) or with decreasing step size (squares) towards the separatrix, the region of strongest changes.

According to the high $\chi_{\mathrm{n}}^{2}$ values, less than 14 spline knots were not sufficient for reasonable modelling. On the other hand, any further increase of the node number did not influence $\chi_{\mathrm{n}}^{2}$ strongly. Also the effect of equally or irregularly distributed nodes on $\chi_{\mathrm{n}}^{2}$ was very small for discharge \#23417. However, the resulting electron temperature profiles differed, as shown on the right-hand side of figure 4 analyzed with 19 nodes each but different node distribution. For both cases, the modelled radiation temperatures 

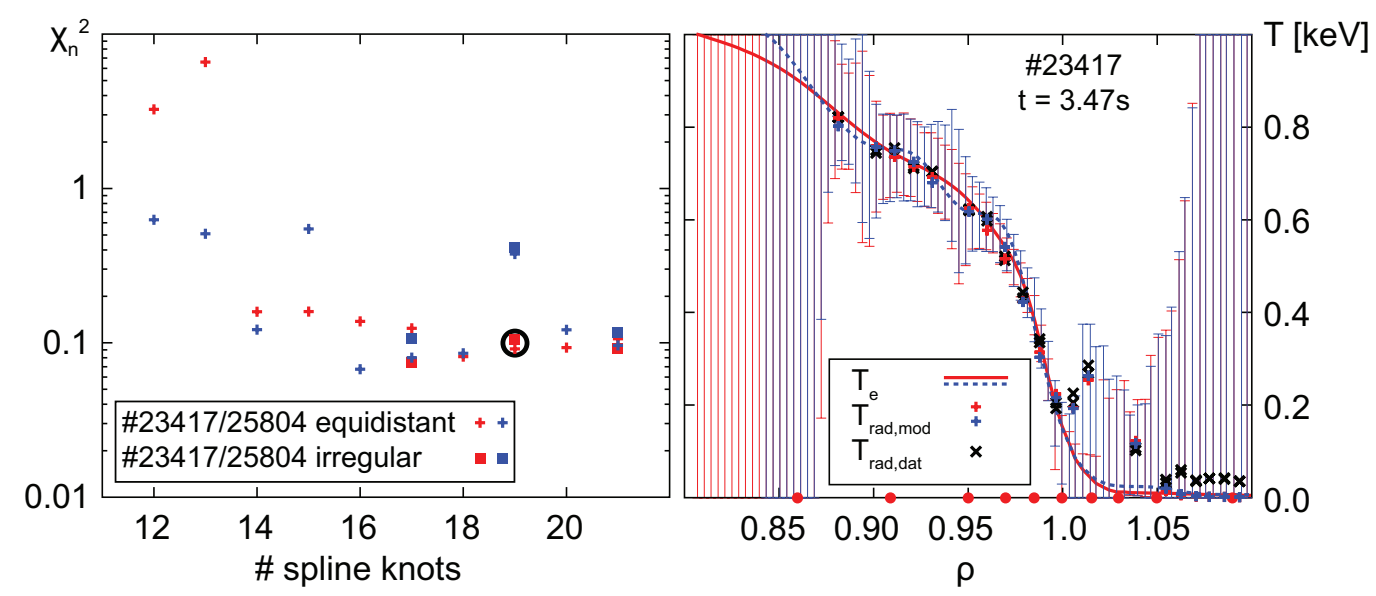

Figure 4. Left: Normalized $\chi^{2}$ depending on the number of spline knots (equally (crosses) or irregularly (squares) distributed) for discharge $\# 25804$ at $t=2.06 \mathrm{~s}$ (blue) and \#23417 at $t=3.47 \mathrm{~s}$ (red), results marked by black circles belong to profiles on the right. Right: Edge $T_{\mathrm{e}}$ profiles obtained with 19 irregularly (red solid line) and equally (blue dashed line) distributed spline knots (irregularly ones indicated by red circles) together with the modelled (red/blue pluses) and measured (black crosses) $T_{\text {rad }}$ values at the positions of their cold resonance for discharge \#23417 at $t=3.47 \mathrm{~s}$

(red/blue pluses) were in good agreement with the measurement (black crosses). Yet, between $\rho=0.85$ and $\rho=0.98$ the electron temperature profile with equally distributed nodes (blue dashed line) exhibited some overshoots in the order of several \%. This well-known phenomena for too much freedom in the spline is called 'ringing'.

\subsection{Sensitivity on the electron density}

Due to the linear electron density dependence of the emissivity (3.3), reasonable ECFM analysis relied on accurate knowledge about the complete electron density profile. The joint analysis of electron density and temperature within the framework of IDA provided the best conditions to deal with the complementary information of these two quantities needed for ECFM. Anyway, electron density estimation is always accompanied by uncertainties in absolute value as well as position. Furthermore, the alignment between electron density and temperature profiles is exposed to uncertainties in the equilibrium reconstruction. The inclusion of the density uncertainties into the ECFM analysis would have greatly increased its complexity and was, therefore, omitted. Instead, the effect of density-scaling both in quantity and position relative to the electron temperature profile on the electron temperature results was investigated in detail.

Absolute value The left plot of figure 5 displays $\chi_{\mathrm{n}}^{2}$ for varied electron density values between $0.8 \times n_{\mathrm{e}}$ and $1.2 \times n_{\mathrm{e}}$ which depicted the upper limit of $20 \%$ uncertainty in the density reconstruction from LIB data. The effect on $\chi_{\mathrm{n}}^{2}$ was very small and also the modelled electron and radiation temperature values were nearly identical. The only outlier for discharge \#25804 with increased electron density of $20 \%$ (cf. blue dashed 

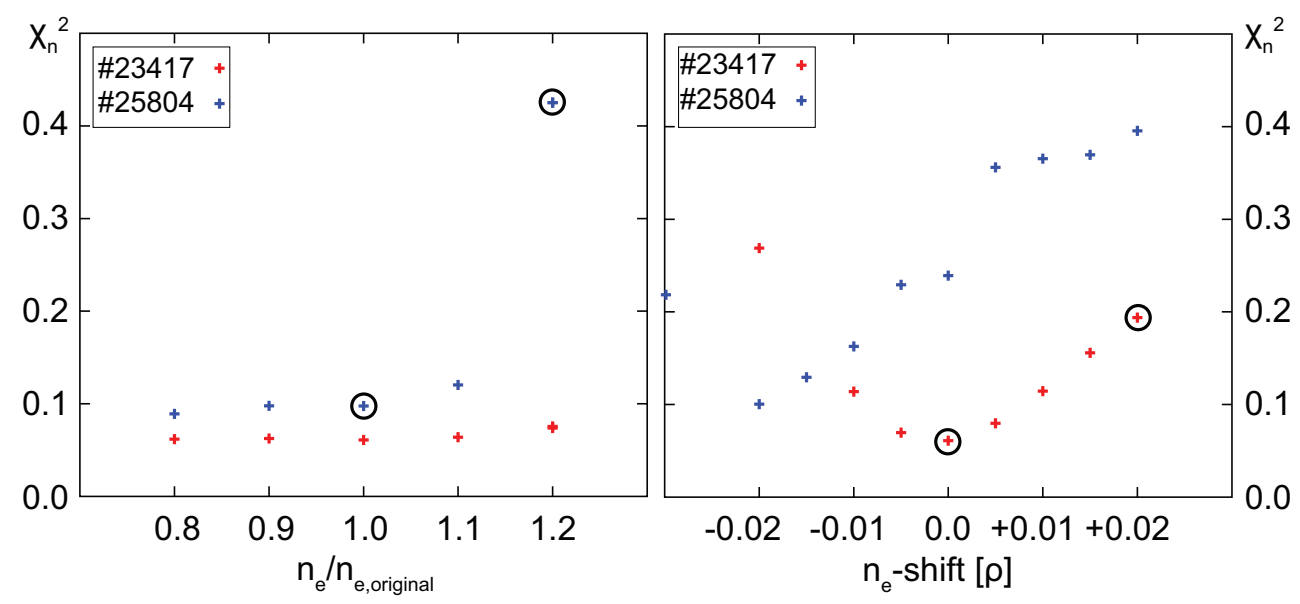

Figure 5. Normalized $\chi^{2}$ depending on the absolute values of $n_{\mathrm{e}}$ (left) and on the alignment of $n_{\mathrm{e}}$ and $T_{\mathrm{e}}$ (right) for discharge \#25804 at $t=2.03 \mathrm{~s}$ (blue) and \#23417 at $t=3.47 \mathrm{~s}$ (red), results marked by black circles belong to profiles in figure 6

line in figure $6(\mathrm{c}))$, resulted from the fact that the cut-off density (black crosses) was exceeded. In this case, the affected ECE channels were set to $T_{\text {rad,mod }}=0$ ( (cf. blue dots in figure 6(a))) and omitted in the analysis by setting their uncertainties to a very large value. This caused a higher value for $\chi_{\mathrm{n}}^{2}$, even if the electron temperature (blue dashed line in a) and the residuals (blue in b) were very similar to the non-scaled (red) case.

Alignment In contrast to the weak influence of the absolute value, an accurate alignment of the electron density profile with the electron temperature profile was of great importance. This can be seen on the right side of figure 5 where $\chi_{\mathrm{n}}^{2}$ is plotted against the electron density shift. Shifts up to $\Delta \rho \pm 0.02$ (+ outwards / - inwards) which correspond to $\Delta R \approx \pm 10 \mathrm{~mm}$ seemed to be reasonable within the uncertainties. For both analyzed discharges $\chi_{\mathrm{n}}^{2}$ exhibited a clear minimum. While for discharge \#23417 the original electron density and temperature profiles were perfectly aligned, the density of $\# 25804$ needed to be shifted towards the temperature profile by $\Delta \rho=-0.02$ to achieve the best modelling results.

The effect of the profile alignment is shown on the right side of figure 6 where the electron density and temperature profiles $(\mathrm{d})$, the line integrated radiation intensities (e) and the corresponding emission and absorption profiles (f) for ECE channel with mean frequency $\nu_{E C E}=111.8 \mathrm{GHz}$ are compared for different applied densities - results obtained with the original electron density profile (black solid line in d) are represented by reddish solid lines, results obtained with the outward shifted electron density profile (black dashed line in d) are represented by bluish dashed lines. The shift resulted in a much higher electron density at the cold resonance position (vertical line at $\rho \approx 1.02$ ) of the selected ECE channel. At this point, the absorption (light blue dashed line in f) was, therefore, strongly increased compared to the original case (orange, solid). To 


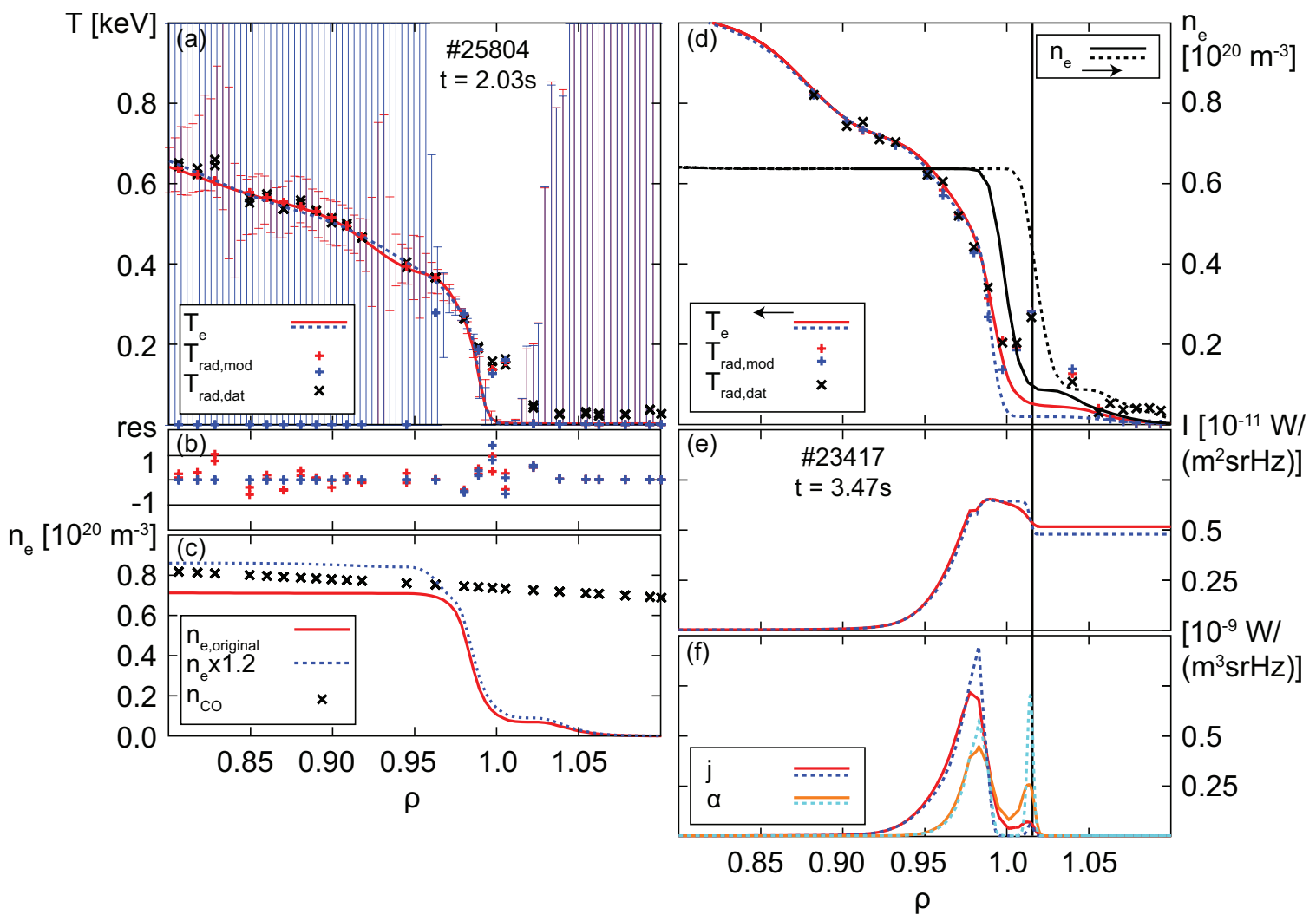

Figure 6. Left: (a) Edge $T_{\mathrm{e}}$ profiles (red/blue lines) together with the modelled (red/blue pluses) and measured (black crosses) $T_{\text {rad values at the positions of their cold }}$ resonance, (b) their residuals and (c) the applied original (red line) and increased (blue line) $n_{\mathrm{e}}$ profile together with the cut-off density (black crosses) of discharge \#25804 at $t=2.03 \mathrm{~s}$. Right: (d) Edge $T_{\mathrm{e}}$ profiles (red/blue lines) together with the modelled (red/blue pluses) and measured (black crosses) $T_{\text {rad }}$ values at the positions of their cold resonance and the original (black solid line) and the shifted (black dashed line) $n_{\mathrm{e}}$ profile, (e) the line integrated radiation intensities and (f) the corresponding emission (red/blue lines) the absorption (orange/light blue lines) profiles of ECE channel with mean frequency $\nu_{E C E}=111.8 \mathrm{GHz}$ for discharge $\# 23417$ at $t=3.47 \mathrm{~s}$

match the ECE measurement anyway, the absorption was reduced in the region around the separatrix $(\rho=0.99-1.01)$ by a decrease in the electron temperature. However, these lower electron temperatures leaded to a misfit between measured (black crosses in d) and modelled (blue pluses) radiation temperatures for the two ECE channels slightly inside the separatrix.

\subsection{Sensitivity on the reflection coefficient}

In optically thin plasma regions multiple ray passes due to reflections increase the measured intensity as described in section 3.3. Unfortunately, an exact modelling with known reflections coefficients was not possible because of the complicated wall structures and resulting diffusive scattering. Instead, a simplified model (3.20) with a parameterized reflection coefficient was used for ECFM analysis. 


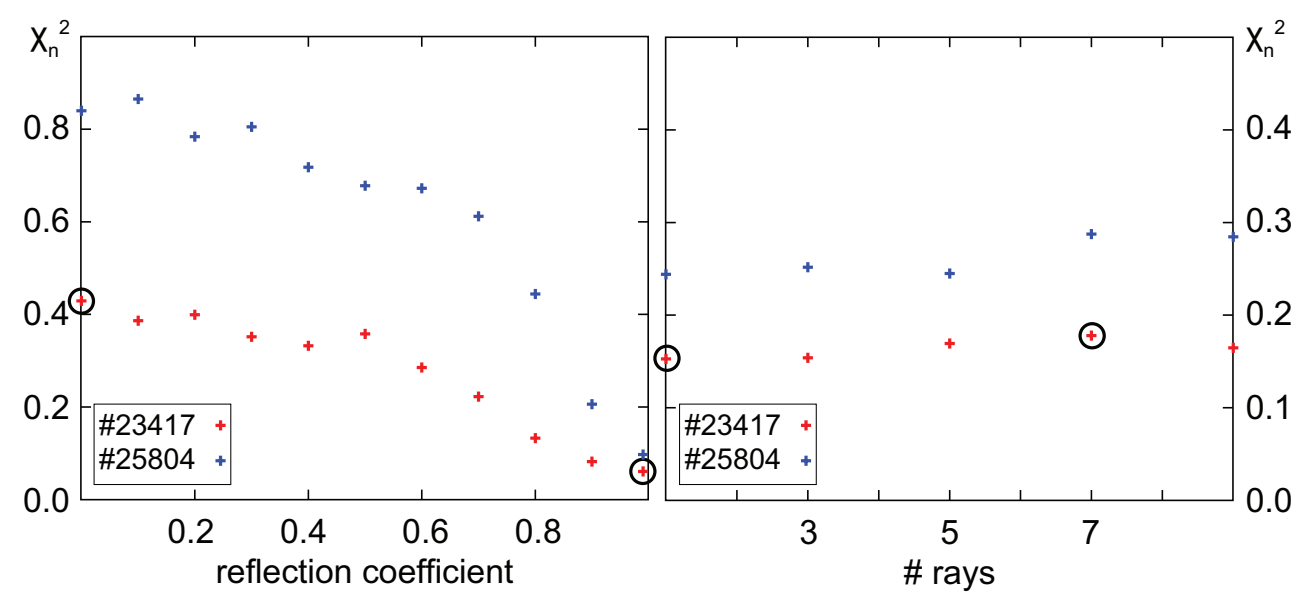

Figure 7. Normalized $\chi^{2}$ depending on the reflection coefficient (left) and on the number of rays (right) for discharge $\# 25804$ at $t=2.03 \mathrm{~s}$ (blue) and $\# 23417$ at $t=3.47 \mathrm{~s}$ (red), results marked by black circles belong to profiles in figure 8

The dependency of $\chi_{\mathrm{n}}^{2}$ on this reflection coefficient is shown on the left side of figure 7. For both discharges, the modelling with highest reflection close to $100 \%$ delivered the best result. For the tungsten coated walls of ASDEX Upgrade this value seemed to be reasonable.

To illustrate the effect of reflections on the radiation intensity, the results for the ECE channel with central frequency of $\nu_{E C E}=111.8 \mathrm{GHz}$ of discharge \#23417 obtained with high reflection coefficient $(R=0.99$, reddish solid lines on the left-hand side of figure 8) were compared to the results obtained without considering reflections (bluish dashed lines). In both cases, the modelled radiation temperature (red/blue pluses in a) for the selected ECE channel matched the measurement (black dot) perfectly. Without reflections, it was directly given by the single-pass intensity (blue dashed line in c). With reflections the single-pass intensity (red solid line in c) was smaller, but reached the same antenna intensity by multiple ray passes. The deviation in the single-pass intensity was produced by a higher electron temperature (red solid line in a) slightly before and at the cold resonance position which caused enhanced absorption (orange solid line in e) in this region.

\subsection{Sensitivity on the antenna pattern}

ECFM analysis incorporates the possibility to account for the finite antenna pattern (cf. section 3.3). The desired number of rays for the discretization of the viewing beam has to be defined in the input file. As already stated, for standard analysis we only consider the central ray, because the computational time increases linearly with the number of rays. This simplification is justified by the small variances in $\chi_{\mathrm{n}}^{2}$ for different ray numbers (cf. right of figure 7 ) and the negligible deviations in the resulting profiles on the right-hand side of figure 8 .

This plot compares the results obtained with the central ray only (reddish, solid) 


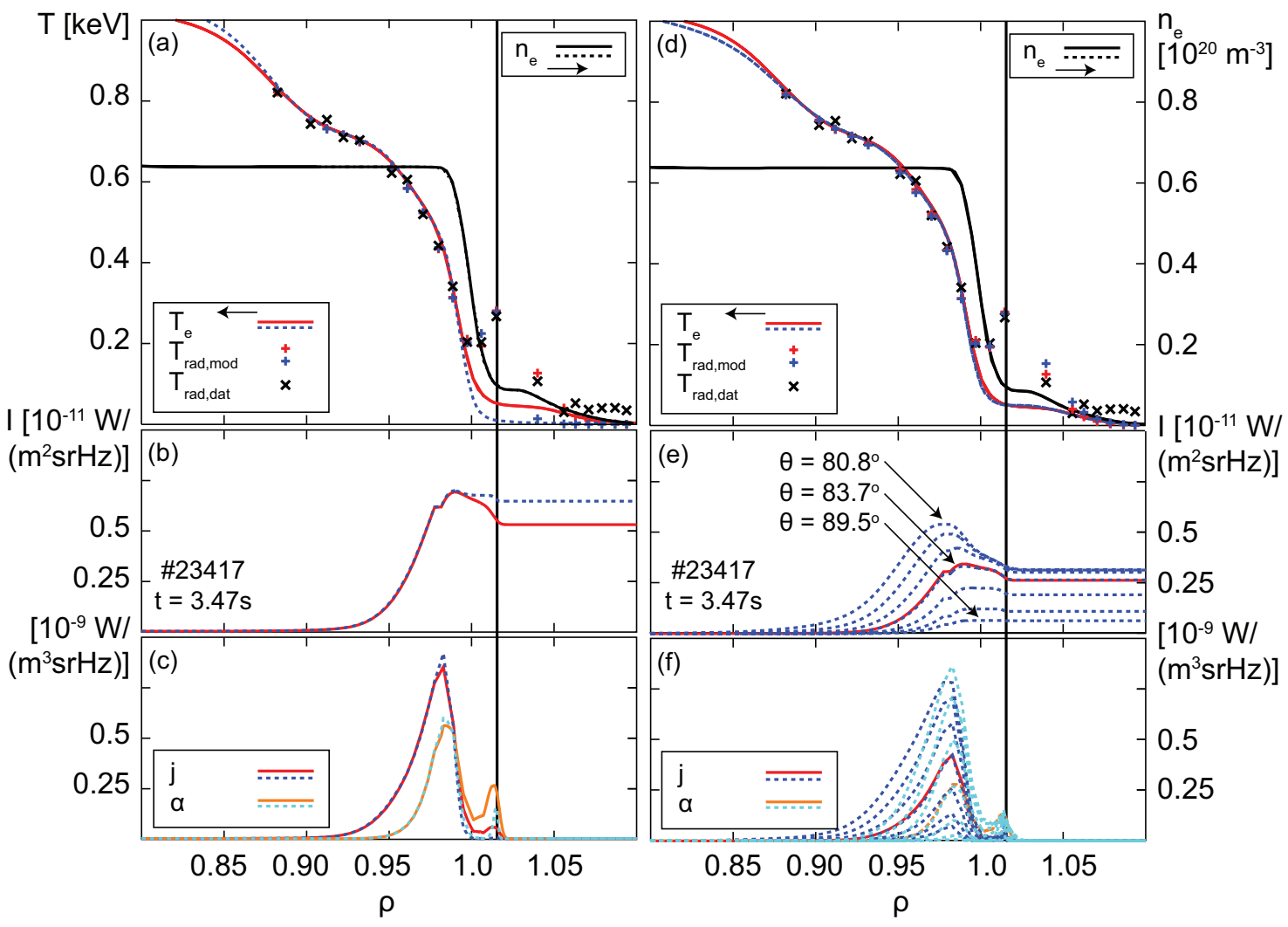

Figure 8. (a,d) Edge $T_{\mathrm{e}}$ profiles with (left) applied reflection of $R=0.99$ (red solid line) and without reflection (blue dashed line) and (right) one ray (red solid line) and seven rays (blue dashed line) together with the modelled (red/blue pluses) and measured (black crosses) $T_{\text {rad }}$ values at the positions of their cold resonance and the applied $n_{\mathrm{e}}$ profile (black line), (b,e) the line integrated radiation intensities (red/blue lines) and (c,f) the corresponding emission (red/blue lines) and absorption (orange/light blue lines) profiles of ECE channel with mean frequency $\nu_{E C E}=$ $111.8 \mathrm{GHz}$ for the only central ray (red line in $\mathrm{f}$ ) and for all seven (unweighted) rays (blue line in $\mathrm{f}$ ) for discharge $\# 23417$ at $t=3.47 \mathrm{~s}$

and with seven rays (bluish, dashed). The electron and modelled radiation temperatures in figure 8(d) and the line integrated intensities (e), emission and absorption (f) profiles for the central rays were almost identical. The emission and absorption profiles of the other rays, seeing the plasma with another angle, of course differed. Anyway, the sum of all rays nearly caused the same result as the central ray. Note here, that the plotted profiles are not yet weighted which means that the actual contributions of the decentral rays were much smaller. An interesting observation is that the ray which was closest to perpendicular viewing exhibited the lowest emission. This is due to the fact, that its shape function was less shifted inwards, because of less Doppler broadening, to hotter and denser plasma regions. 


\section{Validation of the method}

The purpose of this section is to validate our method by demonstrating that it is possible to reconstruct artificial electron density and temperature profiles from the synthetic radiation temperature and by checking the consistency of our electron temperature results with other diagnostics. Furthermore, the limitations of our method is discussed.

\subsection{Reconstruction of artificial data}

The black lines in figure $9(\mathrm{a})$ and (c) show artificially created electron temperature and density profiles. Using these for the modelling of the radiation transport delivers the synthetic ECE data $T_{\text {rad,dat }}$ (black dots in (a)). Reconstructing the input electron temperature by forward modelling of the radiation temperature results in the red profile for the electron temperature and the red dots for the modelled radiation temperature. ECFM was able to reproduce the artificial input profile within the error bars.

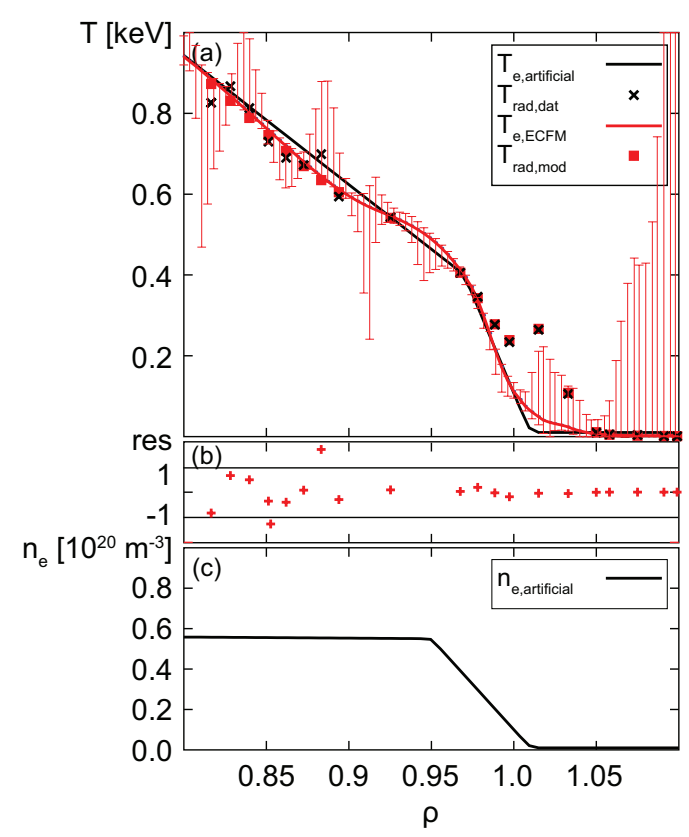

Figure 9. (a) Artificial (black line) and with ECFM analysis reproduced (red line) edge $T_{\mathrm{e}}$ profiles together with the artificial (black crosses) and the modelled (red boxes) $T_{\text {rad }}$ values at the positions of their cold resonance, (b) their residuals and (c) the artificial $n_{\mathrm{e}}$ profile

\subsection{Comparison with other diagnostics}

Depending on the plasma region, there are different methods available at ASDEX Upgrade to get information about the electron temperature. Thomson Scattering spectroscopy (TS) delivers kinetic data for the core and the edge gradient region. From Langmuir probe measurements one can obtain kinetic profiles in the SOL. 
Despite its lower temporal and spacial resolution compared to the ECE measurement, TS diagnostic exhibits the advantage of measuring the electron temperature and density simultaneously and, therefore, delivers important information about the alignment of the profiles. Principles of the ASDEX Upgrade TS system can be found in [16].
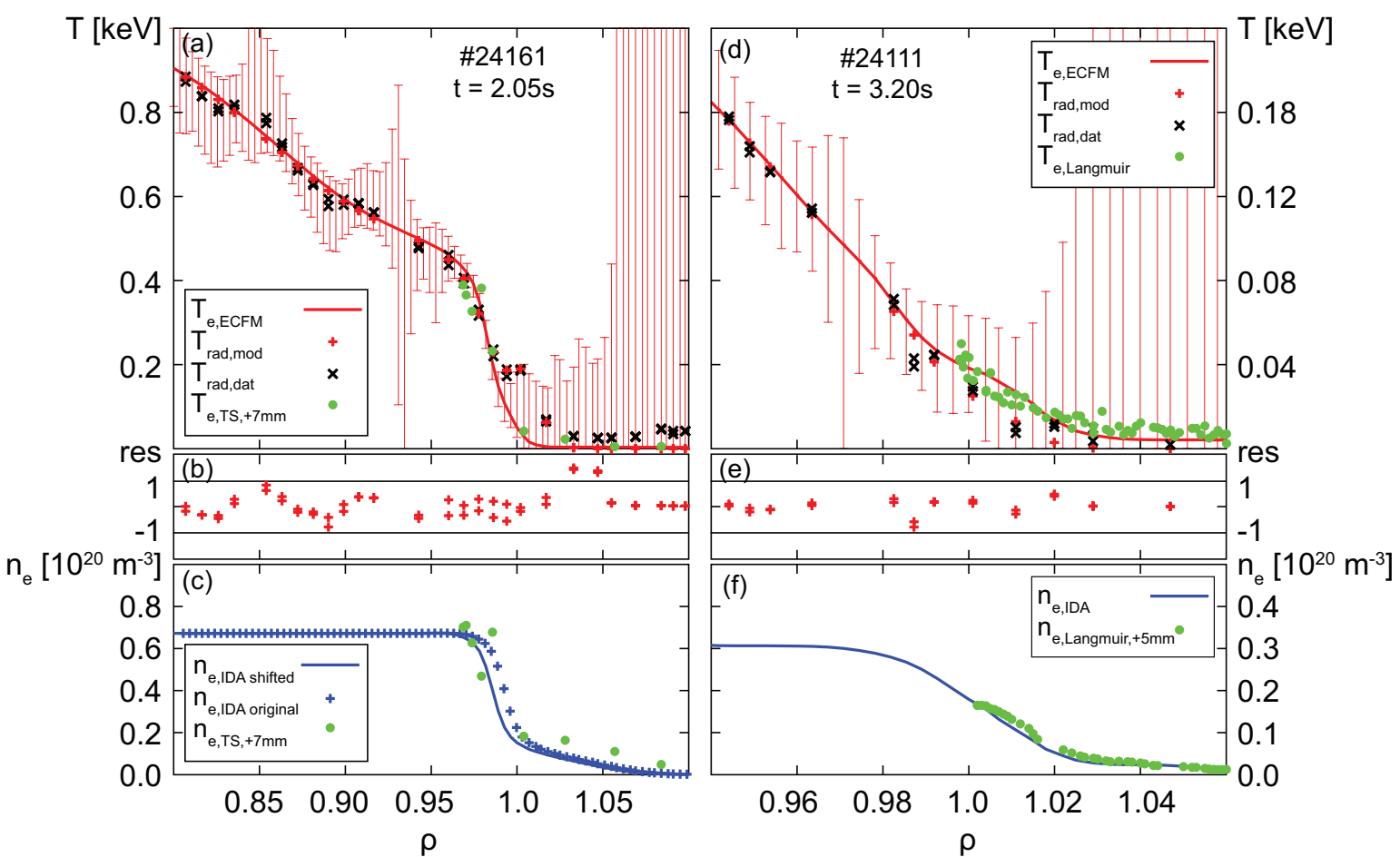

Figure 10. (a,d) Edge $T_{\mathrm{e}}$ profiles from ECFM analysis (red line) and TS (a) or Langmuir probes (d) (green circles) together with the modelled (red pluses) and measured (black crosses) $T_{\text {rad }}$ values at the positions of their cold resonance, (b,e) their residuals and (c,f) the applied (blue line) and original (blue pluses) LIB and TS (a) or Langmuir probe (d) (green circles) $n_{\mathrm{e}}$ profiles of discharge \#24161 at $t=2.04 \mathrm{~s}$ (left) and discharge \#24111 at $t=3.20 \mathrm{~s}$ (right)

The left-hand side of figure 10 shows for discharge \#24161 the electron temperature (a) and density (c) results obtained via ECFM/IDA analysis (red/blue line) compared to the TS data (green circles). After shifting the TS profiles $7 \mathrm{~mm}$ (\#24161) outwards, both, electron temperature and density profiles agreed very well. The shifts were reasonable within the spatial resolution of TS and the uncertainties in the equilibrium reconstruction which acted differently on the line-integrated TS measurement and the magnetic-field dependent localization of the ECE measurement.

The Langmuir probes which can only measure in cold plasma regions deliver electron temperature results in the SOL or in case of an L-mode discharge even up to slightly inside the separatrix. Like TS diagnostics they measure the electron temperature as well as the density, but usually with different probes.

The results of the Langmuir probes on the midplane manipulator for L-mode discharge \#24111 [17] are shown as green circles on the right-hand side of figure 10. 
Without any shift, the electron temperature (d) profile agreed very well with the ECFM result(red line). However, it should be noted that the significance of the electron temperature data from ECE in the SOL was rather low. Outside a certain value (here: $\rho \gtrsim 1.02)$, the fraction of $1^{\text {st }}$ harmonic O-mode contributing to the measured radiation intensity was assumed to dominate over radiation emitted in the $2^{\text {nd }}$ harmonic X-mode and, therefore, the affected ECE channels were omitted in the analysis. This is reflected by the large error bars. The profile in this region was mainly fixed by the wall prior which restricted the electron temperature to values lower than $2 \mathrm{eV}$ at the first wall, as described in section 3.3.

\subsection{Limitations of the model}

As already indicated in section 4, measurements of the electron cyclotron radiation are not sensitive to the electron temperature in the near SOL.

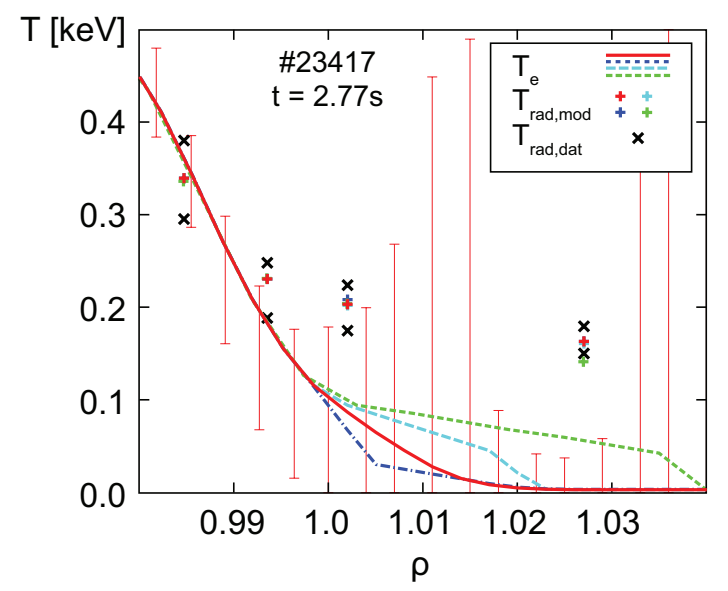

Figure 11. Edge $T_{\mathrm{e}}$ profiles from ECFM analysis (red line) and with different artificially created decay length (coloured lines) together with the modelled (coloured pluses) and measured (black crosses) $T_{\text {rad }}$ values at the positions of their cold resonance of discharge $\# 23417$ at $t=2.77 \mathrm{~s}$

This is demonstrated in figure 11 where the influence of varying the SOL decay length of the electron temperature (coloured dashed lines) compared to the original ECFM result (red solid line, $\lambda_{T_{\mathrm{e}}} \approx 5 \mathrm{~mm}$ ) on the resulting radiation temperature (coloured pluses) was investigated. The steepest SOL profile (blue) thereby corresponded to a decay length of $\lambda_{T_{\mathrm{e}}} \approx 0.5 \mathrm{~mm}$, the flattest SOL profile (green) to $\lambda_{T_{\mathrm{e}}} \approx 20 \mathrm{~mm}$. The small variation of the modelled radiation temperatures compared to scatter in the measurement (black crosses) indicated that it was not possible to resolve these differences in the decay length with ECE. The electron temperature decay length obtained via the power decay length according to (6) in [18] was assumed to lie between $\lambda_{T_{\mathrm{e}}} \approx 8.6 \mathrm{~mm}$ (Spitzer-Härm model) and $\lambda_{T_{\mathrm{e}}} \approx 3.7 \mathrm{~mm}$ (limited-flux model).

Again, the electron temperature profile resulting from ECFM analysis (red line) outside of $\rho=1.02$ was set by the wall prior. In between this position and the separatrix 
the shape was determined by the curvature prior which favoured the least curved profile.

\section{Discussion}

We presented a method to achieve reliable edge electron temperature profiles from ECE data by forward modelling of the radiation transport. Previous 'classical' ECE analysis was not able to exploit the information given by measurements with frequencies resonant in optically thin plasma regions and its edge gradient results mostly relied on extrapolations [9]. The integration of our newly developed ECFM code into the preexisting IDA routine now enables the application of ECE measurements for wellgrounded physics studies based on electron temperature profiles and gradients at the plasma edge.

The most important observation on the electron temperature results obtained by ECFM analysis is that the H-mode edge gradients are actually steeper than the 'classical' ECE analysis would reveal. As expected, the electron temperature around the separatrix and in the near SOL is generally lower than the radiation temperature because radiation from hotter plasma regions is shining through. Furthermore, already in the optically thick gradient and pedestal top regions, the radiation temperature can deviate from the electron temperature at the cold resonance position — in both directions - due to finite emission origin. This implicates that the electron temperature gradients obtained with 'classical' ECE analysis, even if it is done with careful handling of ECE data with respect to the optical depth, can differ significantly from the actual value. Since errors in the temperature are directly reflected in the pressure - a key player for pedestal physics like edge stability - interpretations based on 'classical' ECE analysis might be misleading. It was shown that electron temperature profiles resulting from ECFM analysis are consistent with those from TS measuring the pedestal top and edge gradient region and with those from Langmuir probe measurements around the separatrix.

Another important result is that the 'shine-through' peak can be quantitatively reproduced by ECFM analysis. This phenomena has been unresolved for a long time and was believed to have non-thermal origins [4, 5]. However, according to recent results from JET, the down-shifted emission of thermal electrons in the H-mode edge gradient region can be strong enough to match the cold resonance frequencies of the SOL. Furthermore, due to the low optical depth in the SOL, their emitted radiation actually reaches the antenna [20]. This observation is confirmed by the results from ECFM analysis which is based on a Maxwellian electron distribution and its reliability reflected in the residuals. Anyway, this finding does not allow to exclude the existence of non-thermal electrons. As a consequence of this de-localized radiation origin, the information about the SOL electron temperature is lost, because it is covered by the stronger emission from the edge gradient region. On the other hand, it enhances the resolution of the electron temperature gradient.

We performed a detailed sensitivity study which allowed us to validate the variable program settings and input parameters. Investigations on the influence of the number 
and position of spline knots on the electron temperature results suggest to apply a sufficiently large node number close to the separatrix to be able to resolve the strong gradient, but a rather small number in regions of little changes to avoid 'ringing'. Therefore, we use 21 spline knots with decreasing step size towards the separatrix as standard setting.

Related to the sensitivity on the electron density, we observe that the electron temperature results hardly react on variances of absolute density within its uncertainty range. However, an accurate alignment of electron density and temperature profiles is crucial because the steepness of the electron temperature edge gradient is very sensitive to the profile alignment. On the other hand, the increase of $\chi_{\mathrm{n}}^{2}$ shows that it is not possible to fully compensate an inaccurate electron density position by adapting the electron temperature profile. This means that our method delivers the possibility to estimate the actual relative shift between the ECE and LIB diagnostic from the quality of the fit to the data. We showed that density shifts up to $10 \mathrm{~mm}$ can be necessary to enable a good modelling. However, these shifts are justified within the radial resolution of LIB and the uncertainties in equilibrium reconstruction. Furthermore, our observations concerning the best alignment of the kinetic profiles is consistent with the findings in [13].

Concerning the wall reflections, we observe that the modelling usually works best for almost total reflection. Like the density alignment, the value of the reflection coefficient strongly influences the resulting electron temperature edge gradients. Admittedly, the actual coefficient used for the analysis - which is either chosen manually or selected automatically by the model to enable the best fit to the data - is not based on any physical model or measurement. Anyway, reflections close to $100 \%$, as suggested by our sensitivity study, seems to be reasonable for a tungsten coated wall and small deviances from that do not influence the result significantly.

As discussed in figure 3.3 it is not possible to set up a proper model for possible contributions from $1^{\text {st }}$ harmonic O-mode. However, results obtained with the simple approach described in figure 3.3 showed that it was justified to consider all ECE data with cold resonance within $\rho=1.05$ because the influence of $1^{\text {st }}$ harmonic O-mode contributions within the main plasma, the edge and the near SOL region on the electron temperature result was negligible. Furthermore, it is quite likely that the feature of relatively high and even increasing intensity measured for channels with cold resonance outside $\rho \approx 1.05$ can be explained by contributions from $1^{\text {st }}$ harmonic O-mode.

The occurrence of the 'shine-through' peak should not prevent people from seeing the ECE measurement as a suitable diagnostic for edge analysis. In fact, it really poses a chance. First, because it contains important information about the edge electron temperature gradient. Second, the existence of the 'shine-through' is not itself the reason for the failure of the 'classical' ECE analysis but only the evidence that the optically thick approach fails anyway in the edge region and that one really needs an appropriate model for the electron cyclotron radiation transport and reliable density profiles for well-grounded studies of plasma edge physics based on ECE data. 


\section{Acknowledgments}

M Willensdorfer is a fellow of the Friedrich Schiedel Foundation for Energy Technology.

\section{Appendix. Calculation of the emission shape function}

$$
\begin{aligned}
& \Phi(\omega)=\frac{\zeta^{7 / 2}}{\sqrt{\pi}} \int_{-1}^{1} \mathrm{~d} \beta_{\|} \int_{0}^{\sqrt{1-\beta_{\|}}} \mathrm{d} \beta_{\perp} \beta_{\perp}^{5} \delta\left(\left[1-\beta_{\|} \cos \theta\right] \omega-\frac{\omega_{2 \mathrm{X}}}{\gamma}\right) \times \exp \left(-\zeta\left[\beta_{\perp}^{2}+\beta_{\|}^{2}\right]\right)= \\
& \underbrace{=}_{\mathrm{I}} \frac{\zeta^{7 / 2} \omega}{\sqrt{\pi} \omega_{2 \mathrm{X}}^{2}} \int_{\beta_{\|, 1}}^{\beta_{\|, 2}} \mathrm{~d} \beta_{\|}\left(1-\beta_{\|}^{2}-\left[1-\beta_{\|} \cos \theta\right]^{2} \mu\right)^{2}\left(1-\beta_{\|} \cos \theta\right) \times \\
& \times \exp \left(-\zeta\left[1-\left\{1-\beta_{\|} \cos \theta\right\}^{2} \mu\right]\right)= \\
& \underbrace{=}_{\mathrm{II}} \frac{\zeta^{7 / 2} \omega}{2 \sqrt{\pi} \omega_{2 \mathrm{X}}^{2} \cos \theta} \int_{\alpha_{2}}^{\alpha_{1}} \mathrm{~d} \alpha\left(1-\left[\frac{1-\sqrt{\alpha}}{\cos \theta}\right]^{2}-\alpha \mu\right) \exp (-\zeta[1-\mu \alpha])= \\
& =\ldots= \\
& =\frac{\zeta^{7 / 2} \omega}{2 \sqrt{\pi} \omega_{2 \mathrm{X}}^{2} \cos ^{5} \theta} \int_{\alpha_{2}}^{\alpha_{1}} \mathrm{~d} \alpha\left(\sin ^{4} \theta-4 \sqrt{\alpha} \sin ^{2} \theta+2 \alpha\left[2+\eta \sin ^{2} \theta\right]-4 \eta \alpha^{3 / 2}+\eta^{2} \alpha^{2}\right) \times \\
& \times \exp (-\zeta[1-\mu \alpha])= \\
& \underbrace{=}_{\text {III }} \frac{\zeta^{7 / 2} \omega}{2 \sqrt{\pi} \omega_{2 \mathrm{X}}^{2} \cos ^{5} \theta} \epsilon\left[\operatorname { e x p } ( - \zeta \{ 1 - \mu \alpha \} ) \left(\sin ^{4} \theta+2 \epsilon\left\{\eta \sin ^{2} \theta+2\right\}\left\{\frac{\alpha}{\epsilon}-1\right\}+\right.\right. \\
& \left.\left.+\eta^{2}\left\{\alpha-2 \alpha \epsilon+2 \epsilon^{2}\right\}-4 \eta \alpha^{3 / 2}+4 \sqrt{\alpha}\left\{\frac{3}{2} \eta \epsilon-\sin ^{2} \theta\right\}-4 \sqrt{\epsilon}\left\{\frac{3}{2} \eta \epsilon-\sin ^{2} \theta\right\} F\left(\sqrt{\frac{\alpha}{\epsilon}}\right)\right)\right]_{\alpha_{2}}^{\alpha_{1}}= \\
& \underbrace{=}_{\mathrm{IV}} \frac{\zeta^{3 / 2}}{\sqrt{\pi} \omega \mu \cos ^{5} \theta} \times \\
& \times\left[\exp (-\zeta\{1-\mu \alpha\})\left(-\frac{\sin ^{2} \theta}{\sqrt{\alpha}}+\frac{\eta^{2}}{\zeta \mu}-\frac{1}{\sqrt{\zeta \mu}}\left\{3 \eta-2 \zeta \mu \sin ^{2} \theta\right\} F(\sqrt{\zeta \mu \alpha})\right)\right]_{\alpha_{2}}^{\alpha_{1}}
\end{aligned}
$$

with substitutes: $\mu=\omega^{2} / \omega_{2 \mathrm{X}}^{2}, \quad \eta=1+\mu \cos ^{2} \theta, \quad \epsilon=(\zeta \mu)^{-1}$

I: Integral over $\beta_{\perp}$

$$
\begin{aligned}
& \int_{a}^{b} f(x) \delta(g(x)) d x=\sum_{i} \int_{a}^{b} f(x) \frac{\delta\left(x-x_{i}\right)}{\left|g^{\prime}\left(x_{i}\right)\right|} \mathrm{d} x=\sum_{i} \frac{f\left(x_{i}\right)}{\left|g^{\prime}\left(x_{i}\right)\right|}, \text { where } i \text { are the roots in }[a, b] \\
& \beta_{\|, 1 / 2}=\left(\mu \cos (\theta) \mp \sqrt{1-\mu \sin ^{2} \theta}\right)\left(1+\mu \cos ^{2} \theta\right)^{-1}
\end{aligned}
$$

II: substitution

$\alpha=\left(1-\beta_{\|} \cos \theta\right)^{2} \Rightarrow \mathrm{d} \beta_{\|} / \mathrm{d} \alpha=-(2 \sqrt{\alpha} \cos \theta)^{-1}$ 
III: Dawson integral F

$\int_{x_{1}}^{x_{2}} \mathrm{~d} x x^{3 / 2} e^{x}=\left[x^{3 / 2} e^{x}\right]_{x_{1}}^{x_{2}}-3 / 2 \int_{x_{1}}^{x_{2}} \mathrm{~d} x \sqrt{x} e^{x}=\left[\left(\sqrt{x}-F(\sqrt{x}) e^{x}\right)\right]_{x_{1}}^{x_{2}}$

IV: only higher orders in $\epsilon$

$\epsilon^{0}=0$

$\cos ^{2} \theta=(1-\sqrt{\alpha})^{2} /(1-\mu \alpha)$ (follows from $\left.\epsilon^{0}=0\right)$

\section{References}

[1] Suttrop W et al 1997 Plasma Phys. Control. Fusion 39 2051-66

[2] Hartfuss H J, Geist T and Hirsch M 1997 Plasma Phys. Control. Fusion 39 1693-769

[3] Luhmann N C Jr, Bindsley H, Park H, Sánchez J, Taylor G and Yu C X 2007 Fusion Science and Technology $\mathbf{5 3} 355-96$

[4] Peeters A G, Suttrop W and the ASDEX Upgrade Team 1997 Proc. 10th Joint Workshop on Electron Cyclotron Emission and Electron Cyclotron Resonance Heating (Ameland, Netherlands, 1997) (Singapore: World Scientific Publishing) 403-10

[5] de la Luna E, Sánchez J, Tribaldos V and JET-EFDA contributors 2004 Rev. Sci. Instrum. 75 3831-33

[6] Wang Z, Lohr J, Bell G L, Hsieh C, Luo J, Stockdale R E, Wilgen J B and Zhang J 1995 Proc. 9th Joint Workshop on Electron Cyclotron Emission and Electron Cyclotron Resonance Heating (Borrego Springs, California, 1995) GA-A22038

[7] Pitcher C S, Herrmann A, Murmann H, Reimerdes H, Schweinzer J, Suttrop W, Salzmann H, the ASDEX Upgrade Team and the NBI Group 1997 Plasma Phys. Control. Fusion 39 1129-44

[8] Hutchinson I H 1987 Principles of Plasma Diagnostics (New York: Cambridge University Press) pp $135-50$

[9] Fischer R, Fuchs C J, Kurzan B, Suttrop W, Wolfrum E and the ASDEX Upgrade Team 2010 Fusion Science and Technology $\mathbf{5 8} 675-84$

[10] Fischer R, Dinklage A and Pasch E 2003 Plasma Phys. Control. Fusion 75 1095-111

[11] Dinklage A, Fischer R and Svensson J 2003 Proc. Int. Conf. on Research and Applications of Plasmas (Warsaw, Russia, 2003) I-1.1

[12] Fischer R, Wolfrum E, Schweinzer J and the ASDEX Upgrade Team 2008 Plasma Phys. Control. Fusion 50 085009-37

[13] Schneider P A et al 2012 Differences in H-mode pedestal width of temperature and density (to be published in Plasma Phys. Control. Fusion )

[14] Kallenbach A et al 2004 Plasma Phys. Control. Fusion 46 431-46

[15] Gurnett D A and Bhattacharjee A 2005 Introduction to Plasma Physics (New York: Cambridge University Press) pp 94-127

[16] Kurzan B and Murmann H D 2011 Rev. Sci. Instrum. 82 103501(6pp)

[17] Nold B, Conway G D, Happel T, Müller H W, Ramisch M, Rohde V, Stroth U and the ASDEX Upgrade Team 2010 Plasma Phys. Control. Fusion 52 065005(12pp)

[18] Eich T, Sieglin B, Scarabosio A, Fundamenski W, Goldston R J, Herrmann A and the ASDEX Upgrade Team 2011 Phys. Rev. Lett. 107 215001(4pp)

[19] Stangeby P C, Canik J M and Whyte D G 2010 Nucl. Fus. 50 125003(12pp)

[20] Barrera L et al 2010 Plasma Phys. Control. Fusion 52 085010(14pp) 